\title{
Enhancing comprehensive inversions using the Swarm constellation
}

\author{
Terence J. Sabaka ${ }^{1}$ and Nils Olsen ${ }^{2}$ \\ ${ }^{1}$ Raytheon at Planetary Geodynamics Branch, NASA/Goddard Space Flight Center, Greenbelt, MD 20771, USA \\ ${ }^{2}$ Danish National Space Center, Juliane Maries Vej 30, DK - 2100 Copenhagen Ø, Denmark
}

(Received November 15, 2004; Revised September 2, 2005; Accepted September 28, 2005; Online published April 14, 2006)

\begin{abstract}
This paper reports on the findings of a simulation study designed to test various satellite configurations suggested for the upcoming Swarm magnetic mapping mission. The test is to see whether the mission objectives of recovering small-scale core secular variation (SV) and lithospheric magnetic signals, as well as information about mantle conductivity structure, can be met. The recovery method used in this paper is known as comprehensive inversion (CI) and involves the parameterization of all major fields followed by a co-estimation of these parameters in a least-squares sense in order to achieve proper signal separation. The advantage of coestimation over serial estimation of parameters is demonstrated by example. Synthetic data were calculated for a pool of six Swarm satellites from a model based heavily on the CM4 comprehensive model, but which has more small-scale lithospheric structure, a more complicated magnetospheric field, and an induced field reflecting a 3-D conductivity model. These data also included realistic magnetic noise from spacecraft and payload. Though the parameterization for the $\mathrm{CI}$ is based upon that of CM4, modifications have been made to accommodate these new magnetospheric and induced fields, in particular with orthogonality constraints defined so as to avoid covariance between slowly varying induced fields and SV. The use of these constraints is made feasible through an efficient numerical implementation. Constellations of 4, 3, 2, and 1 satellites were considered; that with 3 was able to meet the mission objectives, consistently resolving the SV to about spherical harmonic (SH) degree $n=15$ and the lithosphere to a limited $n<90$ due to external field leakage, while those with 2 and 1 were not; 4 was an improvement over 3, but was much less than the improvement from 2 to 3 . The resolution of the magnetospheric and induced SH time-series from the 3 satellite configuration was sufficient enough to allow the detection of 3-D mantle conductivity structure in a companion study.
\end{abstract}

Key words: Earth's magnetic field, comprehensive modelling, electromagnetic induction, ionosphere, lithosphere, magnetosphere.

\section{Introduction}

In April 2004 the European Space Agency's (ESA) Living Planet Programme selected Swarm—The Earth's Magnetic Field and Environment Explorers for full implementation under its Earth Explorer mission suite. This mission, tentatively scheduled for launch in 2009 with a four year duration, consists of a magnetic mapping constellation of three satellites in low-Earth, near-polar orbits that will provide simultaneous, high-precision vector measurements at multiple spatial locations (see Friis-Christensen et al., 2006). The specifications of the proposed constellation are for one pair of satellites flying side-by-side in near-polar, circular orbits with an initial altitude and inclination of $450 \mathrm{~km}$ and $86.8^{\circ}$, respectively, separated in the east-west direction by $1^{\circ}-1.5^{\circ}$. The third, higher satellite is proposed to be in a circular orbit with $87.3^{\circ}$ inclination at an initial altitude of $530 \mathrm{~km}$. The differential precession rate between the higher and lower satellite orbital planes is about $2 \mathrm{hr} / \mathrm{yr}$. The resultant sampling of the magnetic field at multiple local times will help to disentangle signals from various magnetic sources and facilitate the resolution of the spatio-temporal

Copyright (C) The Society of Geomagnetism and Earth, Planetary and Space Sciences (SGEPSS); The Seismological Society of Japan; The Volcanological Society of Japan; The Geodetic Society of Japan; The Japanese Society for Planetary Sciences; TERRAPUB aliasing prevalent in single-satellite missions. This will allow Swarm to meet its science objectives in core dynamics and geodynamo processes, lithospheric magnetization, 3-D mantle conductivity, ocean circulation, and magnetic forcing of the upper atmosphere.

During Phase A of the mission, a full end-to-end simulation was performed to test the feasibility of recovering the intended science objectives and to reveal possible design limitations. The simulation consists of two phases: a forward or prediction module and an inverse module. In the forward module, the details of which are given in the companion paper of Olsen et al. (2006), magnetic fields associated with the major near-Earth current systems are synthesized from realistic models at the times and positions of the satellites. The sources considered include the core, crust, magnetosphere, ionosphere, and fields induced in the Earth's outer conducting layers by time-varying external fields. The models that represent the fields from these sources in the most consistent manner are known as Comprehensive Models (CMs) (Sabaka et al., 2002, 2004) and are derived from satellite and observatory data during generally quiet magnetic conditions. Hence, the CMs, particularly CM4 (Sabaka et al., 2004), form the basis of the prediction model used in the forward step, with certain modifications made for application to the Swarm mission. These 
include such things as smaller-scale lithospheric and secular variation (SV) signals, a more complicated magnetospheric field, and an induced field based upon an underlying 3-D conductivity model. In addition, realistic instrument noise has been included in the synthesized output.

The inverse module attempts to solve the inverse problem, that is, attempts to recover the various target fields from the synthesized, noisy data. The target fields in this study include the lithospheric field at all spatial wavelengths, but particularly the small-scale content, the secular variation of the core field, and temporal variations of the broad-scale external and associated induced fields on a scale of hours to weeks. The method of comprehensive inversion $(C I)$ is discussed here, but other approaches contained in this module are covered in companion papers such as Kuvshinov et al. (2006); Maus et al. (2006).

In the CI approach, fields associated with the major near-Earth current systems are parameterized and then coestimated in a weighted least-squares sense; basically the scheme employed in the derivation of the current CMs. As will be seen in Sections 2 and 4.1, this co-estimation is critical to the proper partitioning of signal among sources whose temporal and spatial scales overlap. Conversely, a serial estimation approach, i.e., one in which fields are parameterized and removed in a sequential fashion, can significantly degrade the quality of recovery of the fields. Because the co-estimation procedure modifies each model basis function by actually identifying and removing the effects of all others, it plays a direct role in error analysis, in which case it could be termed "co-accounting". That is, the CI approach has the capability of accounting for unmodelled signal without actually estimating this signal, assuming its functional relationship with the measurements is known. If this relationship involves an infinite number of parameters, then the co-accounting takes place in the data covariance matrix; but for a finite number, it can take place in the parameter space, where it can lead to computationally feasible algorithms since dense data covariance matrices due to non-zero error correlation lengths can be avoided. In this study, data from all local times are analyzed and this means that ionospheric sources must be dealt with; in this case, described by a finite number of parameters. The co-estimation in CI then naturally includes these effects in the error analysis of the fields of interest. Though these ionospheric parameters need not be explicitely solved for, they are in this study in order to check the full range of recoverability in CI.

As for the remainder of this paper, the rationale for applying least-squares co-estimation, i.e., CI, to Swarm will be argued in Section 2. The methodology of applying CI to the simulated Swarm data will be covered in Section 3. This includes modifications to the basic CM4 parameterization used here, which are necessary for describing, in particular, the high-frequency (periods on the order of hours to weeks) magnetospheric and induced portions. The time variations of these fields are discretized into bins during which the fields are considered constant and separability from SV requires that linear equality constraints be incorporated into the least-squares algorithm, which creates some computational issues that will also be discussed. In addition, a brief synopsis of the synthetic data and the selection criteria used in this study will be given. Section 4 discusses the major results of this study, which are the field recovery performances of constellations of various sizes, where it will be shown that the three-satellite constellation described earlier is sufficient to meet the core SV and 3-D induction goals established for Swarm. Of the constellations considered, this one is found to be only marginally worse than its foursatellite counterpart, but significantly better than the twosatellite version. A simple example will also be given which illustrates the superiority of co-estimation over serial estimation in the Swarm context. Finally, a brief description is given of how the magnetospheric and high-frequency induced spherical harmonic time-series were produced from the Swarm simulator data that were later used by Kuvshinov et al. (2006) in detecting simulated 3-D structure in the mantle.

\section{An argument for using least-squares co- estimation with $S$ warm}

The near-Earth magnetic field is a complex superposition of time-varying fields from many different sources whose spatial and temporal scales overlap, especially from the perspective of limited data samplings. Typically a mixture of serial estimation and co-estimation are used to recover fields from certain constituent sources. For example, in order to isolate the lithospheric signal many approaches will first subtract a main field estimate and perhaps estimates of the magnetospheric, ionospheric and induction signals from the data before proceeding (see Arkani-Hamed and Strangway, 1985a,b; Arkani-Hamed and Strangway, 1986; Arkani-Hamed et al., 1994; Cohen and Achache, 1990; Counil et al., 1991; Hamoudi et al., 1998; Ravat et al., 1995; Maus et al., 2002). If, however, some spatial structure of the lithospheric field is obfuscated by these other signals with respect to data observability, then predicted signal power will be erroneously reduced from the resulting lithospheric model due to the serial nature of the process. Although the Swarm constellation configuration will provide unprecedented data coverage at such high precision, which ameliorates some of the problems, it does not address other problems such as poor surface coverage. Furthermore, much is expected of Swarm such that the expected accuracy levels could still be eroded by not correctly treating the signal overlap problem. The co-estimation approach followed in CI takes into account the correlative information between source fields in order to properly partition the signal.

The differences in least-squares co-estimation versus serial estimation can be shown by first considering the solution of the least-squares problem

$$
\min _{\mathbf{x}_{A}, \mathbf{x}_{B}}\left|\mathbf{d}-\left(\begin{array}{ll}
A & B
\end{array}\right)\left(\begin{array}{l}
\mathbf{x}_{A} \\
\mathbf{x}_{B}
\end{array}\right)\right|^{2},
$$

where $|\cdot|$ is the Euclidean norm, $\mathbf{d}$ is the data vector, and $A$ and $B$ are the design matrices associated with two groups of parameters $\mathbf{x}_{A}$ and $\mathbf{x}_{B}$, respectively, which are to be coestimated. The solution to this problem is the solution of the stationary condition, i.e., the normal equations

$$
\left(\begin{array}{ll}
A^{T} A & A^{T} B \\
B^{T} A & B^{T} B
\end{array}\right)\left(\begin{array}{l}
\mathbf{x}_{A} \\
\mathbf{x}_{B}
\end{array}\right)=\left(\begin{array}{l}
A^{T} \mathbf{d} \\
B^{T} \mathbf{d}
\end{array}\right) .
$$


Assuming $\left(A^{T} A\right)^{-1}$ exists, Eq. (2) may be reduced to echelon form

$$
\begin{aligned}
& \left(\begin{array}{cc}
A^{T} A & A^{T} B \\
0 & B^{T} B-B^{T} A\left(A^{T} A\right)^{-1} A^{T} B
\end{array}\right)\left(\begin{array}{l}
\mathbf{x}_{A} \\
\mathbf{x}_{B}
\end{array}\right)= \\
& \left(\begin{array}{c}
A^{T} \mathbf{d} \\
B^{T} \mathbf{d}-B^{T} A\left(A^{T} A\right)^{-1} A^{T} \mathbf{d}
\end{array}\right),
\end{aligned}
$$

where the decoupled equation in $\mathbf{x}_{B}$ may be rewritten as

$$
\begin{gathered}
B^{T}\left(I-A\left(A^{T} A\right)^{-1} A^{T}\right) B \mathbf{x}_{B}= \\
B^{T}\left(I-A\left(A^{T} A\right)^{-1} A^{T}\right) \mathbf{d} .
\end{gathered}
$$

Observe, however, that $I-A\left(A^{T} A\right)^{-1} A^{T}$ is the projection matrix onto the nullspace of the column span of the matrix $A$, and will be denoted as $\mathcal{N}_{A}$. By permuting the parameter space, one can make a completely symmetric argument for the solution of $\mathbf{x}_{A}$ with $\mathcal{N}_{B}$ as the nullspace projector for the columns of $B$. Since these projection matrices are idempotent, the solution to the original least-squares co-estimation problem may be recast as two decoupled problems which lie in each others nullspaces

$$
\left\{\begin{array}{l}
\min _{\mathbf{x}_{A}}\left|\mathcal{N}_{B}\left(\mathbf{d}-A \mathbf{x}_{A}\right)\right|^{2} \\
\min _{\mathbf{x}_{B}}\left|\mathcal{N}_{A}\left(\mathbf{d}-B \mathbf{x}_{B}\right)\right|^{2} .
\end{array}\right.
$$

Note that replacing $\mathcal{N}_{B} \mathbf{d}$ and $\mathcal{N}_{A} \mathbf{d}$ by $\mathbf{d}$ will not alter the solutions, which means that the salient point is the modification to the basis functions, that is, the columns of $A$ and $B$.

A serial estimation of $\mathbf{x}_{A}$ followed by $\mathbf{x}_{B}$ would lead to the following minimization problem for $\mathbf{x}_{B}$

$$
\begin{aligned}
\min _{\mathbf{x}_{B}}\left|\mathcal{N}_{A} \mathbf{d}-B \mathbf{x}_{B}\right|^{2}= & \min _{\mathbf{x}_{B}} \mid \mathcal{N}_{A}\left(\mathbf{d}-B \mathbf{x}_{B}\right)- \\
& \left.\mathcal{R}_{A} B \mathbf{x}_{B}\right|^{2}, \\
= & \min _{\mathbf{x}_{B}}\left(\left|\mathcal{N}_{A}\left(\mathbf{d}-B \mathbf{x}_{B}\right)\right|^{2}+\right. \\
& \left.\left|\mathcal{R}_{A} B \mathbf{x}_{B}\right|^{2}\right),
\end{aligned}
$$

where $\mathcal{R}_{A}=I-\mathcal{N}_{A}$ is the projection matrix onto the range of the column span of $A$ and premultiplication of $\mathbf{d}$ by $\mathcal{N}_{A}$ yields the residuals of $\mathbf{d}$ with respect to parameters $\mathbf{x}_{A}$. Note that the first term in the cost functional in Eq. (7) is identical to that for $\mathbf{x}_{B}$ in the co-estimation problem, but the second term is new; this term, in fact, is a smoothing norm with unit multiplier and will damp, to some extent, the solution in the range of the column span of $A$. However, since $\mathbf{d}_{B}=B \mathbf{x}_{B}$ is the predicted data signal from $\mathbf{x}_{B}$, then one can see that this additional term seeks to minimize its strength over the range of the column span of $A$. In fact, the following length relationships between the data signal vectors predicted by co-estimation, $\mathbf{d}_{B}$, and serial estimation, $\tilde{\mathbf{d}}_{B}$, will be derived in Appendix 1

$$
\left|\mathcal{N}_{A} \tilde{\mathbf{d}}_{B}\right| \leq\left|\tilde{\mathbf{d}}_{B}\right| \leq\left|\mathcal{N}_{A} \mathbf{d}_{B}\right| \leq\left|\mathbf{d}_{B}\right|
$$

where the parameter state vectors from serial estimation, $\tilde{\mathbf{x}}_{B}$, and co-estimation, $\mathbf{x}_{B}$, are given by

$$
\begin{aligned}
& \tilde{\mathbf{x}}_{B}=\left(B^{T} \mathcal{N}_{A} B+B^{T} \mathcal{R}_{A} B\right)^{-1} B^{T} \mathcal{N}_{A} \mathbf{d}, \\
& \mathbf{x}_{B}=\left(B^{T} \mathcal{N}_{A} B\right)^{-1} B^{T} \mathcal{N}_{A} \mathbf{d}
\end{aligned}
$$

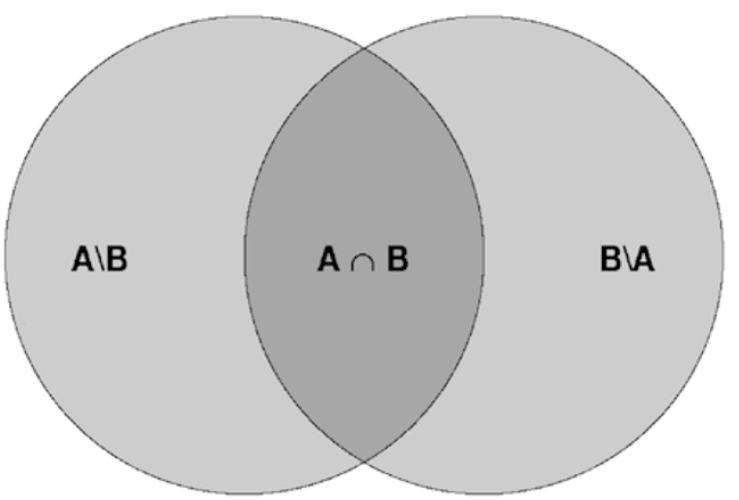

Fig. 1. Venn diagram showing a set-theoretic view of the span of two sets of basis functions ( $A$ and $B$, left and right circles, respectively) in a data space $(D)$ associated with two sets of generic model parameters to be co-estimated in a least-squares sense. Notationally, $A \cap B$ is read " $A$ intersection $B$ ", $A \backslash B$ is read " $A$ minus the intersection of $A$ and $B$ ", and $B \backslash A$ is read " $B$ minus the intersection of $B$ and $A$ ".

Indeed, there will be an artificial reduction in power in a predicted target field if contaminating fields are removed in a separate first step. Notice, however, that this says nothing about the relative lengths of the co-estimated and serial estimated parameter state vectors; intuitively, it seems that the latter should somehow have power artificially removed, but evidently it is estimated such that Eq. (8) holds. In fact, an upper-bound on the ratio of the lengths of $\tilde{\mathbf{x}}_{B}$ to $\mathbf{x}_{B}$ is found in Appendix 1 to be

$$
\frac{\left|\tilde{\mathbf{x}}_{B}\right|}{\left|\mathbf{x}_{B}\right|} \leq \sqrt{\kappa\left(B^{T} \mathcal{N}_{A} B\right)},
$$

where $\kappa\left(B^{T} \mathcal{N}_{A} B\right)$ is the condition number of $B^{T} \mathcal{N}_{A} B$, i.e., the ratio of the largest to smallest eigenvalues of $B^{T} \mathcal{N}_{A} B$ (see Demmel, 1997). Since the condition number is always $\geq 1$, this does not force a reduction in power in $\tilde{\mathbf{x}}_{B}$ with respect to $\mathbf{x}_{B}$. These properites will be illustrated in an example using the Swarm constellation in Section 4.1, and in this case it will be seen that actually $\left|\mathbf{x}_{B}\right|<\left|\tilde{\mathbf{x}}_{B}\right|$.

The rationale for using least-squares co-estimation rather than serial estimation may be further illustrated through settheoretic arguments by considering a data space $D$ in which d and the columns of $A$ and $B$ reside. Let the span of the columns of $A$ and $B$ be represented by circles; the circles then delineate that part of $D$ that may be described by the columns of either $A$ or $B$. By construction, there are four distinct regions: that which may be described by both $A$ and $B$, that is, the intersection of $A$ and $B$ denoted $A \cap B$; that which may be described by $A$, but not $B$, denoted $A \backslash B$; that which may be described by $B$, but not $A$, denoted $B \backslash A$; and that which is described by neither $A$ nor $B$. This is represented visually in the Venn diagram of Fig. 1.

It may be assumed without loss of generality that the partitioned design matrix $(A B)$ is full-rank. Otherwise, there exists some single unobservable parameter represented by $A$ such that $A \backslash B=\oslash$, where $\oslash$ denotes the "empty set", in which case it is simply eliminated and the cycle repeated until $(A B)$ is no longer rank-deficient. If $\mathbf{x}_{A}$ is estimated 
before $\mathbf{x}_{B}$ in a serial approach, then $\mathbf{x}_{A}$ is fit by the entire span of $A$, i.e., the entire circle representing $A$, followed by $\mathbf{x}_{B}$ being fit by the entire span of $B$. However, $A \cap B$ in some sense represents "disputed territory" within which the estimator cannot discriminate between the two overlapping sets. Because $A \cap B$ is not barred from consideration, $\mathbf{x}_{A}$, being the first parameter set, will remove power from this region while $\mathbf{x}_{B}$, finding no power here, will effectively damp this portion of its data space. Note that a reversal in order will necessarily change the solutions.

By contrast, co-estimation will eliminate $A \cap B$ from consideration and rather estimate $\mathbf{x}_{A}$ from $A \backslash B$ and $\mathbf{x}_{B}$ from $B \backslash A$, that is, consider only "undisputed territory", which is logically appealing. The sets $A \backslash B$ and $B \backslash A$ translate into $\mathcal{N}_{B} A$ and $\mathcal{N}_{A} B$, respectively, while $A \cap B$ translates into the span of the columns of $\mathcal{R}_{B} A$ and $\mathcal{R}_{A} B$. It is interesting to note that if $A$ and $B$ represent mutually orthogonal basis functions under a sufficient data distribution, then $A \cap B=$ $\oslash$ and serial and co-estimation will be equivalent.

\section{Methodology}

The justification for a CI paradigm was established in the previous section. The details of its application to Swarm will now be covered, including cases where data have been selected from one to four satellites comprising what is known as Swarm constellation \#2 (Olsen et al., 2006). In addition, parameter modifications to the basic CM4 template and the estimation procedure will be discussed along with computational issues, which were quite formidable.

\subsection{Data}

The Swarm constellation \#2 is actually a pool of six satellite ephemerides of which up to four have been drawn in this study, details of which are provided in Olsen et al. (2006). In the first case, only a single low satellite is used, Swarm A, with initial altitude and inclination of $450 \mathrm{~km}$ and $86.8^{\circ}$, respectively; the second case uses both low-flying satellites, Swarm A and B, differing only in their rightascension of the ascending node (RAAN), resulting in a longitudinal separation of $1.5^{\circ}$; the third case adds a single high-flying satellite, Swarm C, to the mix whose initial altitude and inclination are $550 \mathrm{~km}$ and $87.3^{\circ}$, respectively; and finally, the fourth case adds still another high-flyer, Swarm D, with identical initial altitude and inclination as Swarm C, but with a RAAN of $348^{\circ}$, which separates it by $12^{\circ}$ in longitude from Swarm C, whose RAAN is $0^{\circ}$.

Vector magnetic data were synthesized along the ephemerides every $1 \mathrm{~min}$ from 1997 to 2002 from a prototype of the CM4 model (Sabaka et al., 2004), but with four important modifications: 1) high-degree lithospheric models (up to $n=120$ ) and secular variation models (up to $n=20$ ) were added; 2) the magnetospheric field was determined hourly from a world-wide distribution of groundbased observatories; 3) a 3-D model of electrical conductivity of the crust and mantle was used to compute the induced signal from the magnetosphere (the induced signal from the ionosphere is still synthesized from a 1-D conductivity model); and 4) realistic magnetic noise from spacecraft and payload was added (Olsen et al., 2006). This synthetic noise is based upon CHAMP spacecraft experience and Swarm specifications. It is a random noise that is correlated in time, but uncorrelated among the vector components. The standard deviation of the noise is $(0.07,0.1,0.07) \mathrm{nT}$ for $\left(B_{r}, B_{\theta}, B_{\phi}\right)$, where $(r, \theta, \phi)$, are the usual spherical coordinates, in agreement with $S$ warm performance requirements. Furthermore, the toroidal field from CM4 was not included in the synthesis because it was found to be somewhat unrealistic, especially at the low satellite altitudes; neither an extrapolation of the Ørsted field in radius nor the Magsat field in local time is stable due to insufficient coverage in the variation of these parameters.

The 1997 to 2002 time span was chosen to mimic that portion of the solar cycle through which the mission is scheduled to fly with the intent of producing magnetic conditions similar to those anticipated to be encountered. Two time sampling strategies were used: the first selects $1 \mathrm{~min}$ values at quiet-times only during the 1997-2002 mission envelope, where $t$ is considered quiet when $K_{p}(t)<1^{+}$, $K_{p}(t-3 \mathrm{hr}) \leq 2^{0},\left|D_{\mathrm{st}}\right| \leq 20 \mathrm{nT}$, and $\left|\Delta D_{\mathrm{st}} / \Delta t\right| \leq 3 \mathrm{nT} / \mathrm{hr}$ $(\Delta t=1 \mathrm{hr})$; the second selects all $1 \mathrm{~min}$ values during the 1999-2002 time span, regardless of the magnetic disturbance level. The motivation for the first scheme is to test the recoverability of the core SV signal over the entire mission in the case of a realistically restricted data distribution; the second scheme facilitates the assessment, in the frequency domain, of the transfer function associated with the 3-D conductivity model, which must be computed at equal increments from the resulting model time-series estimates of the magnetospheric and high-frequency (periods substantially shorter than those described by SV) induced fields. After 1999 there is either sufficient separation between the high-low satellite orbital planes or they are at least counter-rotating; two conditions which aid in resolving fine-scale structure. In both schemes, the vector data were generated in the local NEC, i.e., (North, East, Center) coordinate system, implying that vector attitude error has not been considered at this stage.

Ground-based observatory data were omitted from the present study mostly to gauge the effectiveness of the constellation alone in meeting mission requirements and to simplify this initial investigation. It is expected that separability between the static, broad-scale internal field and the static ionospheric baseline field (Sabaka et al., 2002), used to negate this field on the nightside due to diminished conductivity levels, will be degraded somewhat, but this is not expected to unduly affect target field recovery.

\subsection{Parameterization}

Since the essence of the CI is the estimation of parameters associated with an underlying CM, the parameterization used here follows closely that of CM4, combined of course with modifications which address those found in the forward module listed in the previous section. A brief summary of the model parameterization is now given, but detailed descriptions of the portions consistent with CM4 may be found in Sabaka et al. (2002, 2004). The core and lithospheric magnetic fields are expressed as negative gradients of internal potential functions of the form

$$
V_{\mathrm{cl}}(t, \mathbf{r})=\Re\left\{a \sum_{n=1}^{120} \sum_{m=0}^{n}\left(\frac{a}{r}\right)^{n+1} \gamma_{n}^{m}(t) Y_{n}^{m}(\theta, \phi)\right\}
$$


with

$$
Y_{n}^{m}(\theta, \phi)=P_{n}^{m}(\cos \theta) \exp i m \phi
$$

where $a$ is the mean radius of the Earth $(6,371.2 \mathrm{~km}), \mathbf{r}$ is the position vector, $(r, \theta, \phi)$ are the usual geographic spherical polar coordinates, and $Y_{n}^{m}$ and $P_{n}^{m}$ are the Schmidt quasi-normalized surface spherical harmonic and associated Legendre function of degree $n$ and order $m$, respectively (Langel, 1987). The $\Re\{\cdot\}$ operation takes the real part of the expression only. Hence, the $\gamma_{n}^{m}$ are unique complex expansion coefficients, also known as Gauss coefficients. They are related to the usual real Gauss coefficients $g_{n}^{m}$ and $h_{n}^{m}$ according to $\gamma_{n}^{m}=g_{n}^{m}-i h_{n}^{m}$. A degree truncation level of 120 is used, which is commensurate with the signal content from this source in the data.

As in CM4, cubic B-splines describe the time variations in $\dot{\gamma}_{n}^{m}(t)$, and hence, the underlying core SV. In this study, the epoch is taken at the synthetic mission mid-point, 1999.5, which is also the position of the single interior knot. This configuration is the same for all Gauss coefficients of $n \leq 20$; above $n=20$ all coefficients are considered constant. This gives a total of 16,840 real parameters describing the core and lithospheric fields.

The ionospheric field is assumed to be due to currents flowing in a sheet at $h=110 \mathrm{~km}$ altitude, and so is represented as the negative gradient of potential functions above and below the sheet, across which the radial field is continuous. Since the conductivity pattern governing the current flow is highly organized about the ambient magnetic field, special harmonic basis functions are employed which possess this symmetry via their dependence upon the Quasi-dipole (QD) coordinate system (Richmond, 1995). Since the currents are ultimately driven by thermal winds due to variable atmospheric heating, these QD functions are mostly sun-synchronous, but include some faster and slower modes to account for main field interactions. Further solar dependence is built in as seasonal fluctuation and a rigid expansion-contraction response to solar radio flux. Although the dominant morphology of the quiet, regular (Sq) portion of the ionospheric field is due to the broad-scale, coupled $\mathrm{Sq}$ vortices in the northern and southern hemispheres, there is a significant signal associated with the narrow, sinuous equatorial electrojet (EEJ), which is present on the dayside along the dip equator. Therefore, the required QD latitudinal resolution is much higher than in longitudinal (or magnetic local time in the sun-synchronous frame).

The induced contributions are assumed due to an a priori four layer, 1-D, radially varying conductivity model derived from $\mathrm{Sq}$ and $D_{\text {st }}$ data at selected European observatories (Olsen, 1998). Responses with seasonal or longer periods are derived by assuming that the mantle is an insulator in the region $a-\delta \leq r \leq a$ and superconducting in $r<a-\delta$, where $\delta=1000 \mathrm{~km}$ and corresponds to periods longer than about a week.

This information is quantified in the following parameterizations in $\epsilon_{k s p}^{l}$ for the ionospheric potentials in the regions $a \leq r \leq a+h$ (included for completeness even though surface data were not used in this study) and $r>a+h$, where the "," indicates the latter region,

$$
\begin{aligned}
V_{\text {ion }}\left(t, t_{\mathrm{mut}}, \mathbf{r}\right)= & \Re\left\{\sum_{s, p, l, k} \epsilon_{k s p}^{l} \sum_{n, m}\right. \\
& {\left[\left(d_{k n}^{l m}\right)^{*} S_{n s p, \mathrm{e}}^{m}\left(t, t_{\mathrm{mut}}, \mathbf{r}\right)+\right.} \\
& \left.\left.\left(f_{k n s p}^{l m}\right)^{*} S_{n s p, \mathrm{i}}^{m}\left(t, t_{\mathrm{mut}}, \mathbf{r}\right)\right]\right\}, \\
V_{\mathrm{ion}}^{\prime}\left(t, t_{\mathrm{mut}}, \mathbf{r}\right)= & \Re\left\{\sum_{s, p, l, k} \epsilon_{k s p}^{l} \sum_{n, m}\right. \\
& {\left[\left(g_{k n}^{l m}\right)^{*}+\left(f_{k n s p}^{l m}\right)^{*}\right] } \\
& \left.S_{n s p, \mathrm{i}}^{m}\left(t, t_{\mathrm{mut}}, \mathbf{r}\right)\right\},
\end{aligned}
$$

with

$$
\begin{aligned}
S_{n s p, \mathrm{e}}^{m}\left(t, t_{\mathrm{mut}}, \mathbf{r}\right)= & a\left(\frac{r}{a}\right)^{n} P_{n}^{m}\left(\cos \theta_{\mathrm{d}}\right) \\
& \exp i\left(m \phi_{\mathrm{d}}+\omega_{s} s t+\omega_{p} p t_{\mathrm{mut}}\right), \\
S_{n s p, \mathrm{i}}^{m}\left(t, t_{\mathrm{mut}}, \mathbf{r}\right)= & a\left(\frac{a}{r}\right)^{n+1} P_{n}^{m}\left(\cos \theta_{\mathrm{d}}\right) \\
& \exp i\left(m \phi_{\mathrm{d}}+\omega_{s} s t+\omega_{p} p t_{\mathrm{mut}}\right),
\end{aligned}
$$

where the asterisk denotes complex conjugation, $\left(r, \theta_{\mathrm{d}}, \phi_{\mathrm{d}}\right)$ are dipole spherical polar coordinates, $t$ is universal time (UT) in years, $t_{\text {mut }}$ is magnetic universal time (MUT) in hours, whose reference meridian is the prime of the dipole coordinate system, and $s$ and $\omega_{s}$ are the seasonal wavenumber and fundamental frequency of 1 cycle/yr, respectively, while $p$ and $\omega_{p}$ are the diurnal wavenumber and fundamental frequency of 1 cycle/24 hrs, respectively. Summations over $s$ reflect annual and semi-annual seasonal variability while those over $p$ reflect the first four dominant subharmonics (periods as short as $6 \mathrm{hrs}$ ). Summations over $l$ and $k$ are designed to maintain a constant resolution level in QD latitude of about $4^{\circ}$ for each $p$ value. The $d_{k n}^{l m}$ are coefficients of spherical transforms used in representing the harmonic QD function of degree $k$ and order $l$ as linear combinations of the usual spherical harmonics of degree $n$ and order $m$ in dipole coordinates; the $n$ and $m$ summations reflect the required truncations levels. The $g_{k n}^{l m}$ are similar to $d_{k n}^{l m}$, but they also contain information about the radial continuity of the ionospheric field across the sheet current. The $f_{\text {knsp }}^{l m}$ contain information about the QD symmetry of the internal induced fields, and accordingly, information about the transfer function mappings from ionosphere to induced imposed by the 1-D conductivity model; hence, the dependence upon $s$ and $p$. The $g_{k n}^{l m}$ and $f_{k n s p}^{l m}$ matrices allow for a complete representation of ionospheric primary fields above and below the sheet currents and secondary induced fields in Earth's outer conducting layers in terms of one set of ionospheric primary potential parameters, of which there are 5,520 .

\subsubsection{Magnetospheric and high-frequency induced} fields

The parameterization of the magnetospheric and associated high-frequency induced fields in this study represents a significant departure from current $\mathrm{CM}$ approaches. The idea here is to discretize these fields in time by dividing the mission envelope into contiguous, short-duration bins in which static models of both the magnetospheric and induced fields are estimated. This is in part motivated by the 


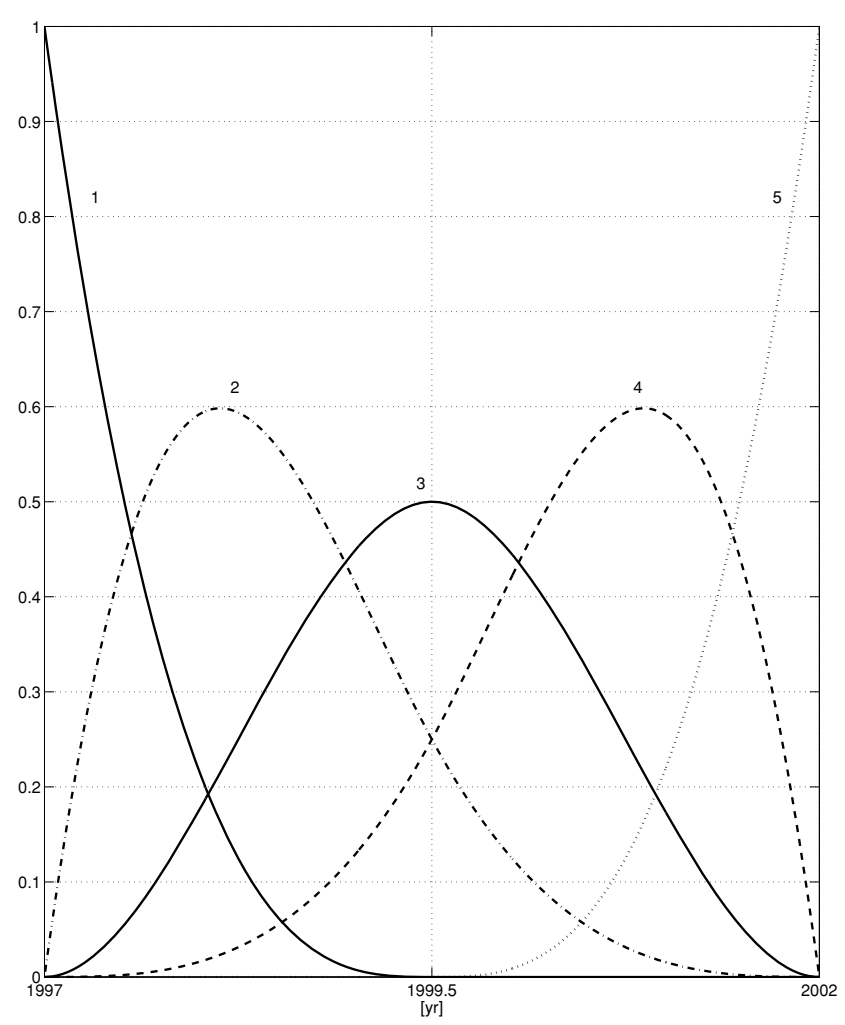

Fig. 2. Schematic of the cubic B-spline distribution over the mission envelope used to represent the secular variation of each main field Gauss coefficient. There is a single interior knot at 1999.5 and 4 exterior knots at each endpoint 1997 and 2002. The functions are numbered sequentially from earliest to latest.

unprecedented coverage in local time at a given UT afforded by the Swarm constellation. It also has the advantage of eliminating the dependence upon the $D_{\text {st }}$ index in tracking ring current variability, which is known to have shortcomings such as baseline shifting, etc. (Olsen et al., 2005), and reducing the dependence on a priori conductivity models. This latter point opens the possibilities of actually deducing information on conductivity structure from Swarm.

With current sources located outside of the sampling region, these two fields may be represented in the $j$ th bin again as the negative gradient of potentials of the form

$$
\begin{aligned}
V_{\mathrm{mag}, \mathrm{j}}(\mathbf{r})= & \Re\left\{a \sum_{n=1}^{N_{\max }} \sum_{m=0}^{\min \left(n, M_{\mathrm{mxm}}\right)}\left(\frac{r}{a}\right)^{n}\right. \\
& \left.\mu_{n, j}^{m} Y_{n}^{m}\left(\theta_{\mathrm{d}}, \phi_{\mathrm{d}}\right)\right\}, \\
V_{\text {ind, }}(\mathbf{r})= & \Re\left\{a \sum_{n=1}^{N_{\max }} \sum_{m=0}^{\min \left(n, M_{\mathrm{mxi}}\right)}\left(\frac{a}{r}\right)^{n+1}\right. \\
& \left.\iota_{n, j}^{m} Y_{n}^{m}\left(\theta_{\mathrm{d}}, \phi_{\mathrm{d}}\right)\right\},
\end{aligned}
$$

where $N_{\max }$ is the degree truncation level for both field expansions, and $M_{\mathrm{mxm}}$ and $M_{\mathrm{mxi}}$ are the order truncation levels for the magnetospheric and induced expansions, respectively. The truncation levels of these expansions are influenced, as will be seen in Section 4.2, by the bin duration and the configuration of the constellation.

Let $\Delta T$ be the bin duration and $T_{a}$ and $T_{b}$ be the beginning and ending times of the mission envelope where the number of bins is given by $\ell=\left(T_{b}-T_{a}\right) / \Delta T$ and the $j$ th bin, $\mathcal{B}_{j}$, is defined as $\mathcal{B}_{j} \equiv\left[T_{a}+(j-1) \Delta T, T_{a}+j \Delta T\right)$ or $\mathcal{B}_{\ell} \equiv\left[T_{a}+(\ell-1) \Delta T, T_{a}+\ell \Delta T\right]$. Comparing Eqs. (12) and (19), one sees that in function space on $T=\left[T_{a}, T_{b}\right]$

$$
\gamma_{n}^{m} \in \lim _{\Delta T \rightarrow 0} \operatorname{Sp}\left(\iota_{n, j}^{k}, j=1, \ldots, \ell, k=0, \ldots, n\right),
$$

where $\operatorname{Sp}(\cdot)$ denotes the span of a set and $\iota_{n, j}^{k}$ is treated as a constant function on $\mathcal{B}_{j}$. Indexing over $k$ is necessary since $\gamma_{n}^{m}$ and $\iota_{n, j}^{k}$ are with respect to two different coordinate systems, geographic and dipole, respectively, and spherical harmonics of degree $n$ form an irreducible representation of the rotation group (Cohen-Tannoudji et al., 1977). Thus, at some $\Delta T$, separation of the $\iota_{n, j}^{m}$ from $\gamma_{n}^{m}$ will become problematic since the former set becomes an increasingly more accurate discretization of the latter with decreasing $\Delta T$. To remedy the situation, constraints are sought which will enforce some form of orthogonalization between the $\iota_{n, j}^{m}$ and $\gamma_{n}^{m}$ over the data sampling times, and thus, temporally decorrelate the two fields pointwise in space. Mutual adjustment of both sets of parameters in the estimation could lead to a highly non-linear optimization problem, and so the high-frequency induced field is made subordinate to the main field. That is, the main field temporal structure is permitted to freely adjust while the induced field is relegated to fit the remaining high-frequency perturbations superimposed on the former. These perturbation periods would be shorter than the resolving period of the main field basis functions, which are integrals of cubic Bsplines, i.e., quartic polynomials within the knot intervals. This is physically reasonable given that there are four polynomial roots over 30 months intervals leading to regions of uniform sign on the order of 6 mo in duration in $\gamma_{n}^{m}(t)$, which is taken as the resolving period, and this is shorter than variations expected to be seen from core processes due to mantle screening. Thus, the $\iota_{n, j}^{m}$ are expected to capture most of the induced field due to the highly variable magnetospheric field. An obvious caveat is the false attribution of long-period induced signals to the core SV, which are likely manifestations, for instance, of the solar cycle. However, the hope is that real information about the conductivity structure will be gleaned for shorter periods.

Formally, the orthogonality conditions are introduced by defining an inner product, $<,>_{D}$, on the space of data sampling times such that for two arbitrary functions $f$ and $g$ defined on $T$

$$
\begin{aligned}
<f, g>_{D} & \equiv \int_{T} f(\tau) g(\tau) \delta\left(\Pi_{k=1}^{N_{\ell}}\left(\tau-t_{k}\right)\right) d \tau, \\
& =\sum_{k=1}^{N_{\ell}} f\left(t_{k}\right) g\left(t_{k}\right),
\end{aligned}
$$

where $\delta(\cdot)$ is the Dirac delta function and $N_{j}$ is the number of data sampling times, $t_{k}$, residing in bins $\mathcal{B}_{1}$ through $\mathcal{B}_{j}$, with $N_{0} \equiv 0$ (which is needed later). Thus, $<,>_{D}$ reduces to a vector dot product discretized at the data sampling times. In order to enforce orthogonality in time with any main field pointwise in space, the inner product of each set $\left\{\iota_{n, j}^{m}, j=1, \ldots, \ell\right\}$ with each main field basis function 
$b_{i}(t)$ must vanish. In this study, the $\mathrm{SV}$ is represented by cubic B-splines with one interior knot at 1999.5 and 4 exterior knots at each endpoint 1997 and 2002. Each cubic spline occupies 4 adjacent knot intervals giving a total of 5 functions in this case. Figure 2 illustrates the cubic Bspline distribution and numbers the 5 functions sequentially from earliest to latest. For the main field this means 6 basis functions, including a static baseline, at most for any given Gauss coefficient. If the number of real, high-frequency induced coefficients in a bin is $L_{\mathrm{bi}}=M_{\mathrm{mxi}}\left(M_{\mathrm{mxi}}+2\right)+$ $\left(N_{\max }-M_{\mathrm{mxi}}\right)\left(2 M_{\mathrm{mxi}}+1\right)$, then the number of linear, homogeneous constraints that must be imposed is $K=$ $6 L_{\mathrm{bi}}$. The form of the constraint corresponding to the $i$ th main field basis function and the induced coefficient of degree $n$ and order $m$ is

$$
<b_{i}, \iota_{n}^{m}>_{D} \equiv \sum_{j=1}^{\ell} \iota_{n, j}^{m} \sum_{k=N_{j-1}+1}^{N_{j}} b_{i}\left(t_{k}\right) .
$$

The constraints may be assembled into a matrix equation of the form

$$
G_{\mathrm{i}} \iota=\mathbf{0},
$$

where $\iota$ is the vector of all high-frequency induced field expansion coefficients of length $L_{\mathrm{i}}=\ell L_{\mathrm{bi}}$ and $G_{\mathrm{i}}$ is the $K \times L_{\mathrm{i}}$ constraint matrix. In the equally-incremented selection scheme, if $N_{\max }=3, M_{\mathrm{mxm}}=1$ and $M_{\mathrm{mxi}}=3$ for $1 \mathrm{hr}$ bins over the three year sampling interval, then $\ell=26,304$, $L_{\mathrm{bi}}=15, L_{\mathrm{i}}=394,560, L_{\mathrm{bm}}=9, L_{\mathrm{m}}=236,736$ (analogous to $L_{\mathrm{bi}}$ and $L_{\mathrm{i}}$, respectively, but for the magnetosphere), and $K=90$; thus, a total of $L_{\mathrm{mi}}=631,296$ real coefficients describe the magnetospheric and high-frequency induced fields.

\subsection{Linear least-squares with linear equality con- straints}

The CI employs weighted least-squares to estimate the model parameter state, $\mathbf{x}$, and in this study, incorporates linear equality constraints, $G_{\mathrm{i}}$, to enforce pointwise temporal decorrelation between the high-frequency induced field and the core SV field. Because only vector measurements are used, the problem is linear in the model parameters. Therefore, the linear least-squares problem with linear equality constraints (LSLE) may be written as

$$
\operatorname{LSLE}\left\{\begin{array}{c}
\min _{\mathbf{x}}|\mathbf{d}-A \mathbf{x}|^{2} \\
\text { subject to } G \mathbf{x}=\mathbf{0}
\end{array},\right.
$$

where, $\mathbf{d}$ is again the data vector, $A$ is the design matrix associated with $\mathbf{x}$, and $G$ is a sparse matrix whose nontrivial portion corresponds to $G_{\mathrm{i}}$. Data are weighted according to $\sin \theta$ such that the resulting distribution is more uniform, which is especially important for polar orbiting satellites. This weighting is understood to have been applied to $\mathbf{d}$ and $A$ in Eq. (25) as a premultiplication by the appropriate $\sqrt{\sin \theta}$ and will not be discussed further. The solution to LSLE is found directly from the Lagrange multiplier theorem (Bertsekas, 1995; Golub and Van Loan, 1989; Toutenburg, 1982)

$$
\mathbf{x}=\left[E^{-1}-E^{-1} G^{T}\left(G E^{-1} G^{T}\right)^{-1} G E^{-1}\right] \mathbf{y},
$$

where

$$
\begin{aligned}
& E=A^{T} A, \\
& \mathbf{y}=A^{T} \mathbf{d} .
\end{aligned}
$$

It can be seen that the solution in Eq. (26) indeed satisfies the constraint portion of Eq. (25), as required.

Previous CMs derived from POGO, Magsat, Ørsted, and CHAMP satellite data and from observatory data (Sabaka et al., 2002, 2004) have required additional regularization, particularly in the SV and ionospheric portions of the models. This is in part due to inadequacies in data quality and distribution either geographically or in local time. This regularization is usually introduced as additional quadratic smoothing terms (terms whose minima correspond to $\mathbf{x}=$ 0) in the least-squares cost function. However, these terms are omitted here in order to investigate whether the quality and distribution of Swarm data is satisfactory to resolve all parameters, with the exception of those mentioned earlier. It is expected, however, that reality will be more complicated than this simulation and these regularizations will very well be necessary in selecting the best models.

3.3.1 Computational issues Unless there is a compelling need, it is generally not recommended that the system matrix be explicitely inverted when solving a linear set of equations. Rather, it is more efficient to solve the system through iterative methods or through matrix factorization methods such as an "LU" decomposition. However, Eq. (26) is cast in the form of an explicit matrix inversion since (Sorenson, 1980)

$$
\begin{aligned}
\lim _{\lambda \rightarrow \infty}\left(E+\lambda G^{T} G\right)^{-1}= & E^{-1}-E^{-1} G^{T} \\
& \left(G E^{-1} G^{T}\right)^{-1} G E^{-1},
\end{aligned}
$$

which suggests that a more efficient factorization method may exist.

Consider a partitioning of $\mathbf{x}$ such that $\mathbf{x}_{B}$ are the parameters affecting data only in a particular bin, i.e., the magnetospheric and high-frequency induced parameters, and $\mathbf{x}_{C}$ are the parameters whose effects are common to all data, i.e., the core, lithospheric, ionospheric and associated induced parameters. Let $\mathcal{G}$ be that portion of $G$ corresponding to $\mathbf{x}_{B}$ such that $G=\left(\begin{array}{ll}\mathcal{G} & 0\end{array}\right)$. Problem LSLE may alternatively be restated in terms of Lagrange multiplier theory (Bertsekas, 1995; Golub and Van Loan, 1989; Toutenburg, 1982) as

$$
\begin{gathered}
\operatorname{LSLE}\left\{\operatorname { m i n } _ { \mathbf { x } _ { B } , \mathbf { x } _ { C } } \left(\left|\mathbf{d}-\left(\begin{array}{ll}
A_{B} & A_{C}
\end{array}\right)\left(\begin{array}{c}
\mathbf{x}_{B} \\
\mathbf{x}_{C}
\end{array}\right)\right|^{2}+\right.\right. \\
\left.\lim _{\lambda \rightarrow \infty} \lambda\left|\mathcal{G} \mathbf{x}_{B}\right|^{2}\right),
\end{gathered}
$$

where $A_{B}$ and $A_{C}$ are appropriate partitions of $A$ and $\lambda$ is the Lagrange multiplier for the constraint equations. The solution of LSLE is given by the stationary condition

$$
\lim _{\lambda \rightarrow \infty}\left(\begin{array}{cc}
E_{B B}+\lambda \mathcal{G}^{T} \mathcal{G} & E_{B C} \\
E_{B C}^{T} & E_{C C}
\end{array}\right)\left(\begin{array}{l}
\mathbf{x}_{B} \\
\mathbf{x}_{C}
\end{array}\right)=\left(\begin{array}{l}
\mathbf{y}_{B} \\
\mathbf{y}_{C}
\end{array}\right),
$$

where $E_{B B}=A_{B}^{T} A_{B}, E_{B C}=A_{B}^{T} A_{C}, E_{C C}=A_{C}^{T} A_{C}$, $\mathbf{y}_{B}=A_{B}^{T} \mathbf{d}$, and $\mathbf{y}_{C}=A_{C}^{T} \mathbf{d}$. Applying Eq. (29) to 

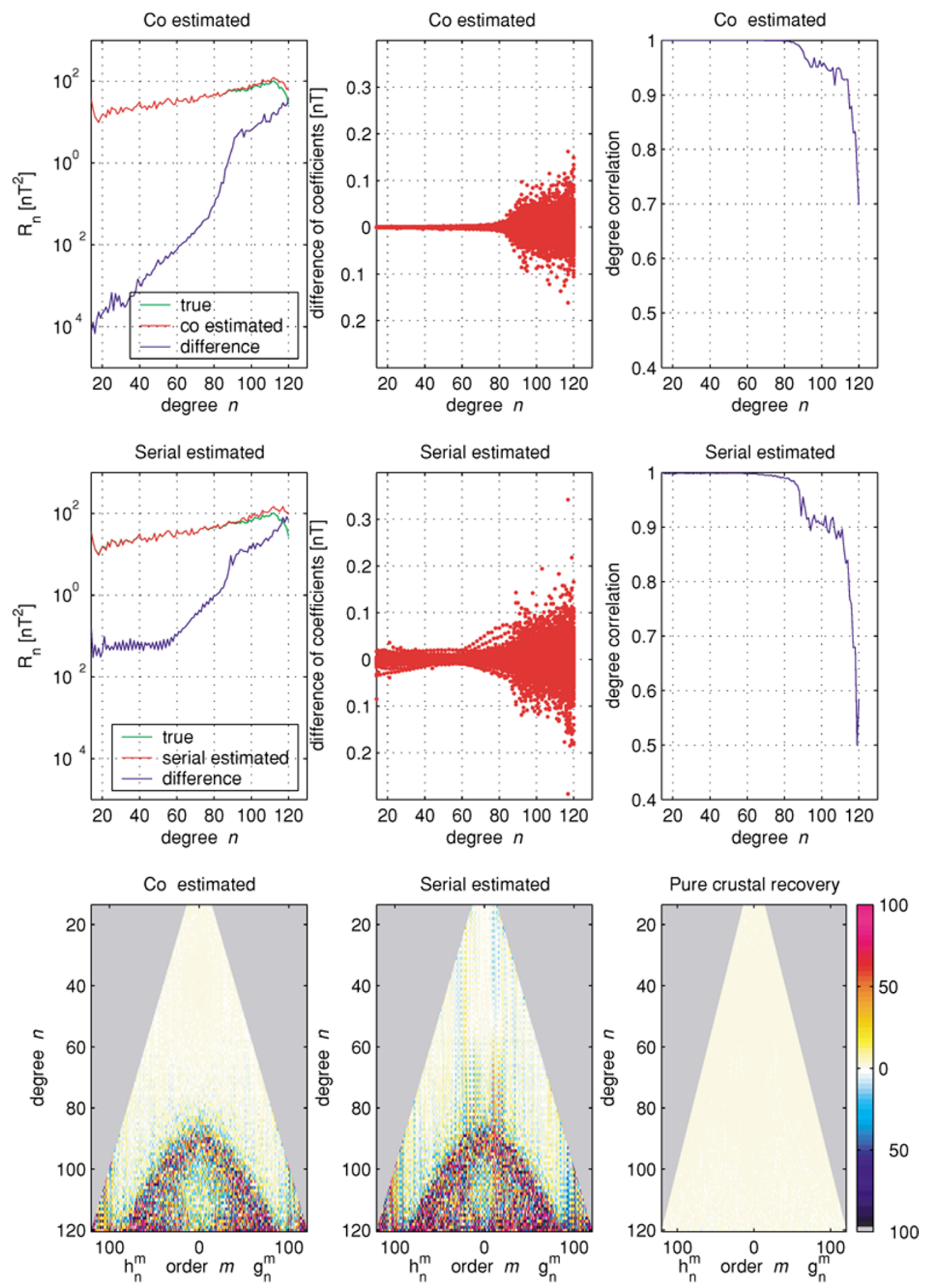

Fig. 3. Comparison of true and recovered crustal field coefficients at Earth's surface from SW-ABCD data containing both crustal, from $n=14-120$, and magnetospheric contributions using both co-estimation and serial estimation schemes. For the top two tiers, the left are the $R_{n}$ spectra for the true (green), recovered (red) and for the difference between true and recovered (blue) crustal models. In the middle are the differences in coefficients as a function of degree $n$. On the right are the degree correlations, $\rho_{n}$, between the recovered and true models. The bottom tier shows the sensitivity matrices, $S(n, m)$, of the crustal fields recovered by co- (left) and serial (middle) estimation and when the SW-ABCD data contained only a crustal contribution (right).

$\lim _{\lambda \rightarrow \infty}\left(E_{B B}+\lambda \mathcal{G}^{T} \mathcal{G}\right)^{-1}$ allows for a relatively straight forward block-Cholesky factorization to be performed sequentially by bin. This is because the $L_{\mathrm{mi}} \times L_{\mathrm{mi}}$ matrix $E_{B B}$ has a sparse block-diagonal structure due to the temporally local nature of the $\mathbf{x}_{B}$ parameters. There is one symmetric $L_{\mathrm{b}} \times L_{\mathrm{b}}$ block for each bin, where $L_{\mathrm{b}}=L_{\mathrm{bi}}+L_{\mathrm{bm}}$. Since $\mathcal{G}$ may be easily partitioned across bins, the $\mathcal{G} E_{B B}^{-1} \mathcal{G}^{T}$ matrix can be easily accumulated sequentially by bin. This is a great savings over the formation of $G E^{-1} G^{T}$ since $E^{-1}$ is necessarily dense.

The algorithm then requires one pass through the data for the block-Cholesky factorization phase and a possible second pass (depending on whether bin-oriented matrices are stored during the first pass) for the back-substitution phase. If $L_{\text {cli }}$ is the length of $\mathbf{x}_{C}$, which in this study is 22,360 , then the storage requirements for the lower-triangular por- 
tion of a matrix of order $L$ plus one right-hand-side vector are $L(L+3) / 2=252,574,049$ words, where $L=$ $L_{\text {cli }}+L_{\mathrm{b}}+K=22,474$.

Actual computation was done on halem, the HP/Compaq AlphaServer Super-Cluster located at the NASA Center for Computational Sciences at Goddard Space Flight Center. This is a large-scale, distributed-memory, parallel computer. Linear algebraic operations were performed on 64 processors using the ScaLAPACK software as well as the Parallel Basic Linear Algebra Subprograms, (PBLAS) and the Basic Linear Algebra Communication Subroutines (BLACS). Additional information may be found at http://www.netlib.org/scalapack.

\section{Results and Discussion}

In order to assess the quality of recovery of the target fields, three different metrics will be used:

Difference in spectra If $g_{n}^{m}$ and $h_{n}^{m}$ are the real Gauss coefficients of the internal field expansion, then the meansquared field magnitude at Earth's surface from degree $n$, $R_{n}$, known as the Lowes-Mauersberger spectrum (Langel, 1987), is given by

$$
\begin{aligned}
R_{n} & =(n+1) \sum_{m=0}^{n}\left[\left(g_{n}^{m}\right)^{2}+\left(h_{n}^{m}\right)^{2}\right], \\
& =(n+1)\left|\mathbf{g}_{n}\right|^{2},
\end{aligned}
$$

where $\mathbf{g}_{n}$ is the vector of spherical harmonic coefficients of degree $n$. Hence, $R_{n}$ is rotationally invariant, and these spectra, as well as those of the model differences, i.e., $\Delta \mathbf{g}_{n}$, gauge the agreement in magnitude of the true and recovered $\mathbf{g}_{n}$.

Degree correlation To gauge the agreement in direction between two vectors, $\mathbf{g}_{n, 1}$ and $\mathbf{g}_{n, 2}$, of spherical harmonic coefficients of degree $n$, the degree correlation, $\rho_{n}$, is computed as

$$
\begin{aligned}
\rho_{n}= & \frac{\sum_{m=0}^{n}\left[g_{n, 1}^{m} g_{n, 2}^{m}+h_{n, 1}^{m} h_{n, 2}^{m}\right]}{\sqrt{\sum_{m=0}^{n}\left[\left(g_{n, 1}^{m}\right)^{2}+\left(h_{n, 1}^{m}\right)^{2}\right]}} \\
& \times \frac{1}{\sqrt{\sum_{m=0}^{n}\left[\left(g_{n, 2}^{m}\right)^{2}+\left(h_{n, 2}^{m}\right)^{2}\right]}} \\
= & \frac{\mathbf{g}_{n, 1} \cdot \mathbf{g}_{n, 2}}{\left|\mathbf{g}_{n, 1}\right|\left|\mathbf{g}_{n, 2}\right|}, \\
= & \cos \zeta_{n},
\end{aligned}
$$

where $\zeta_{n}$ is the angle between $\mathbf{g}_{n, 1}$ and $\mathbf{g}_{n, 2}$. Recovery is deemed satisfactory whenever $\rho_{n} \geq 0.7$ or roughly $\zeta_{n} \leq$ $45^{\circ}$, i.e., the vectors are more correlated than uncorrelated.

Sensitivity matrix To assess the agreement of two models for a particular coefficient, a sensitivity matrix, $S(n, m)$, is constructed in which coefficient differences are normalized by the mean spectral amplitude for degree $n$. If $k_{n}^{m}$ is the recovered Gauss coefficient such that $k_{n}^{m}=g_{n}^{m}$ for $m \geq 0$ and $k_{n}^{m}=h_{n}^{-m}$ for $m<0$, and $c_{n}^{m}$ is the corresponding true coefficient, then

$$
S(n, m)=100 \frac{k_{n}^{m}-c_{n}^{m}}{\sqrt{\frac{1}{2 n+1} \sum_{m=-n}^{n}\left(c_{n}^{m}\right)^{2}}} .
$$

\subsection{Recovery using co- versus serial estimation}

In Section 2 it was argued that a recovery approach based upon co-estimation of the parameters should be superior to one using serial estimation. In this section, an example is provided which illustrates this in the context of Swarm where only the magnetospheric and static $n=14-120$ degree lithospheric contributions are taken from the data of the four Swarm satellites A, B, C and D, denoted constellation SW-ABCD, selected during quiet-time using the criteria described earlier. Instrument noise is not considered here. This yields 789,763 vector observations per satellite for a total measurement count of $\operatorname{dim}(\mathbf{d})=9,477,156$. These are analyzed over $1 \mathrm{hr}$ bins with magnetospheric expansions defined by $N_{\max }=3$ and $M_{\mathrm{mxm}}=1$. This corresponds to the hourly analysis of world-wide observatories used to synthesize this data mentioned earlier. In addition, a single Swarm satellite traverses roughly $2 / 3$ of an orbit per hour, which should be sufficient for resolving these truncation levels. This results in $L_{\mathrm{bm}}=L_{\mathrm{b}}=9$ coefficients per $1 \mathrm{hr}$ bin. The number of bins having a sufficient amount of data for this parameterization is $\ell \approx 14,000$. Thus, the total number of magnetospheric coefficients is about 126,000 . If a degree $n=1-120$ internal static expansion is used to model the lithosphere, then a grand total of $\operatorname{dim}(\mathbf{x}) \approx 140,640$ parameters will be estimated. These parameters are both co-estimated and serial estimated; the latter estimating and removing the magnetospheric contributions from the data first and then estimating the lithospheric field separately. The data weighting is as described in Section 3.3 .

The resulting quality of recovery of the crustal coefficients from these two approaches is illustrated in Fig. 3. The $R_{n}$ spectra, coefficient differences and degree correlations all show the degraded nature of the serial recovery with respect to co-estimation. This is especially true for the low to mid-degree range where the dispersion of the serial estimate from the true model is over two orders of magnitude higher in $R_{n}$ than for the co-estimate. It is suspected that this is the likely range of the parameter space intersection between magnetospheric and lithospheric basis functions. In fact, a test case was attempted in which an additional static $n=1-13$ degree lithospheric contribution was retained in the data, but while this was successful for co-estimation, it failed completely for serial estimation, thus emboldening the low-degree intersection argument. The most direct comparision is seen in the sensitivity matrices which show spurious excursions in the serial estimate along columns of equal order $m$ which do not appear in the co-estimate for $n<65$.

The Euclidean lengths of the various projections of predicted crustal signal vectors in Eq. (8) have been computed and are listed in Table 1, along with those for the crustal 
Table 1. Euclidean lengths of crustal parameter state vectors, $\mathbf{x}_{B}$, and various projections of predicted crustal signal vectors, $\mathbf{d}_{B}$, derived from SW-ABCD data containing both crustal, from $n=14-120$, and magnetospheric contributions using both co-estimation and serial estimation schemes. Here, $A$ and $B$ denote the magnetosphere and crust, respectively. Units are in $\mathrm{nT}$.

\begin{tabular}{ccc}
\hline & Co-estimated & Serial estimated \\
\hline$\left|\mathbf{x}_{B}\right|$ & 8.47 & 8.63 \\
$\left|\mathcal{R}_{A} \mathbf{d}_{B}\right|$ & 779.49 & 637.28 \\
$\left|\mathcal{N}_{A} \mathbf{d}_{B}\right|$ & $5,269.21$ & $5,183.12$ \\
$\left|\mathbf{d}_{B}\right|$ & $5,326.55$ & $5,222.15$ \\
\hline
\end{tabular}
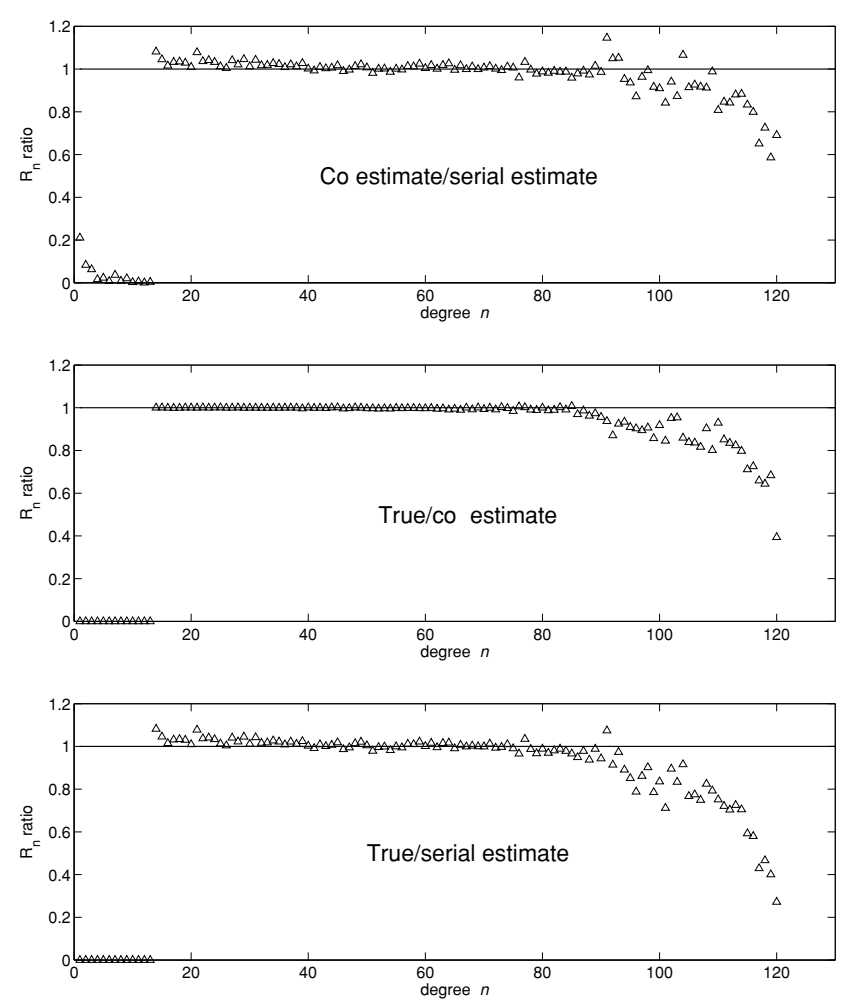

Fig. 4. Ratios of crustal $R_{n}$ values at Earth's surface per degree from the true, co-estimated, and serial estimated models. Top shows co-estimated to serial estimated, middle shows true to co-estimated, and bottom shows true to serial estimated ratios.

parameter state vectors, as derived from co-estimation and serial estimation. Indeed, all of the predicted signal lengths are in accordance with Eq. (8). In addition, the projections onto the range of the magnetospheric column span also show decreased power in the serial estimation case; something intuitively satisfying, but not necessarily required. What is very interesting, however, is that the length of the serial estimate of the crustal parameter vector exceeds that of the co-estimate. Recall from Eq. (11) of Section 2 that the upper-bound on the ratio of the former to the latter is always $\geq 1$. Evidently, additional structure is needed in $\mathbf{x}_{B}$ in this case to accommodate the second term in the cost function of Eq. (7).

In order to investigate this further, plots were made of the ratios of crustal $R_{n}$ values at Earth's surface per degree from the true, co-estimated, and serial estimated. While one might consider this a comparison of predicted signals, the cancellation of the $n+1$ terms in Eq. (33) leaves a simple ratio of parameter variances per degree. The top, middle, and bottom plots in Fig. 4 show the co-estimated to serial estimated, true to co-estimated, and true to serial estimated ratios. Focusing on the $n=14-75$ regime reveals an erroneous decrease in parameter strength for the serial estimate, which is not seen in the co-estimate. For $n>80$, both estimates have excessive power, but even more so for the serial estimate; apparently the high degree terms account for the longer length of the serial estimate. For $n<14$, the true model has zero power, but the top plot shows that the serial estimate has significantly more power than the co-estimate.

While this case study shows the superior recovery capability of co-estimation over serial estimation, it does also reveal a problem with the current general analysis which is manifested as a prominent "hemispherical" excursion pattern for $n>85$ in the sensitivity matrices of both approaches. To investigate this, another test was done in which only the static $n=14-120$ degree lithospheric contribution in the SW-ABCD data was retained. This was analyzed directly using a spherical harmonic expansion of like truncation level in order to see if the pattern was due to some type of sampling inadequacy. The resulting matrix at the lower-right of Fig. 3 shows near perfect recovery and indicates that no such sampling problem exists. Since the pattern exists even after correction of the crustal basis functions for parameterized magnetospheric effects, as attested to in the co-estimation plot, this suggests that the time-varying magnetospheric field is not being entirely explained by the parameterization used here. If the magnetosphere were static throughout the mission and modelled as such, then one would expect this field to be nearorthogonal, due to possible sampling issues, to the static lithosphere. However, this is a case in which a portion of the unmodelled, time-varying external field appears as a static internal field in the null space of the magnetospheric model; a space shared by both the co-estimation and serial estimation. The actual shape, and degree/order range of the excursion pattern is likely related to the orbital inclination angles of the SW-ABCD satellites. In fact, difference plots between the vector components of the true crustal field and those predicted from co-estimation and serial estimation (not included here) reveal structure along the orbits. This type of contamination is common when dealing with data concentrated along tracks and is usually addressed by well-known methods such as levelling (see Green, 1983; Luyendyk, 1997; Minty, 1991), although see the companion paper of Maus et al. (2006) for an alternative approach in crustal field extraction from Swarm. It is beyond the scope of this paper to pursue the problem any further here and it will be left for future research. However, this will necessarily limit the maximum degree of resolution of the crustal field in this study to about $n=90$.

\subsection{Recovery from quiet-time data}

4.2.1 Swarm A, B, C and D A selection of data from the SW-ABCD constellation identical to that of Section 4.1 was considered here except that now the data contain all source contributions, including instrument noise. Again, these were analyzed over $1 \mathrm{hr}$ bins with magnetospheric ex- 

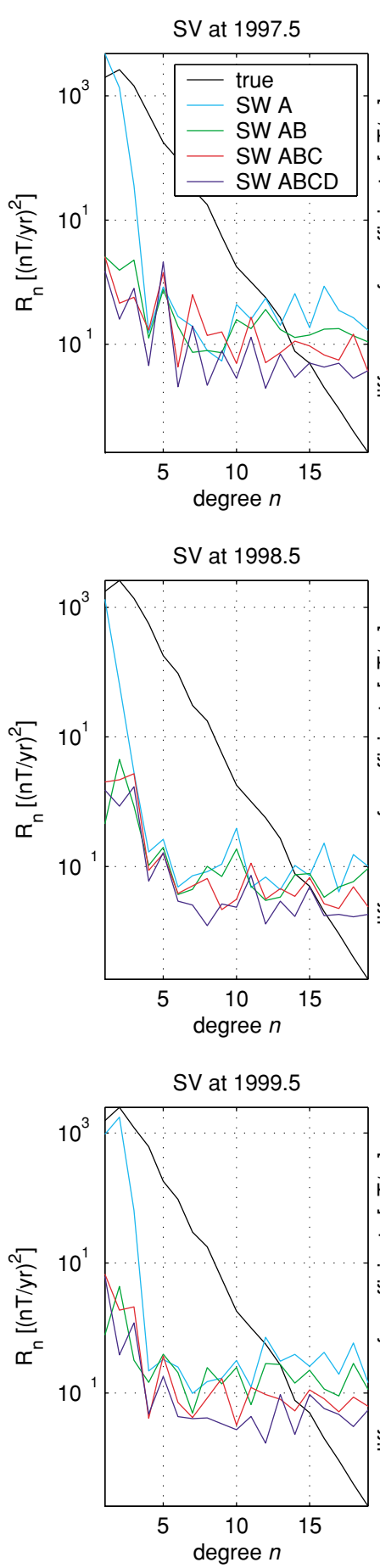
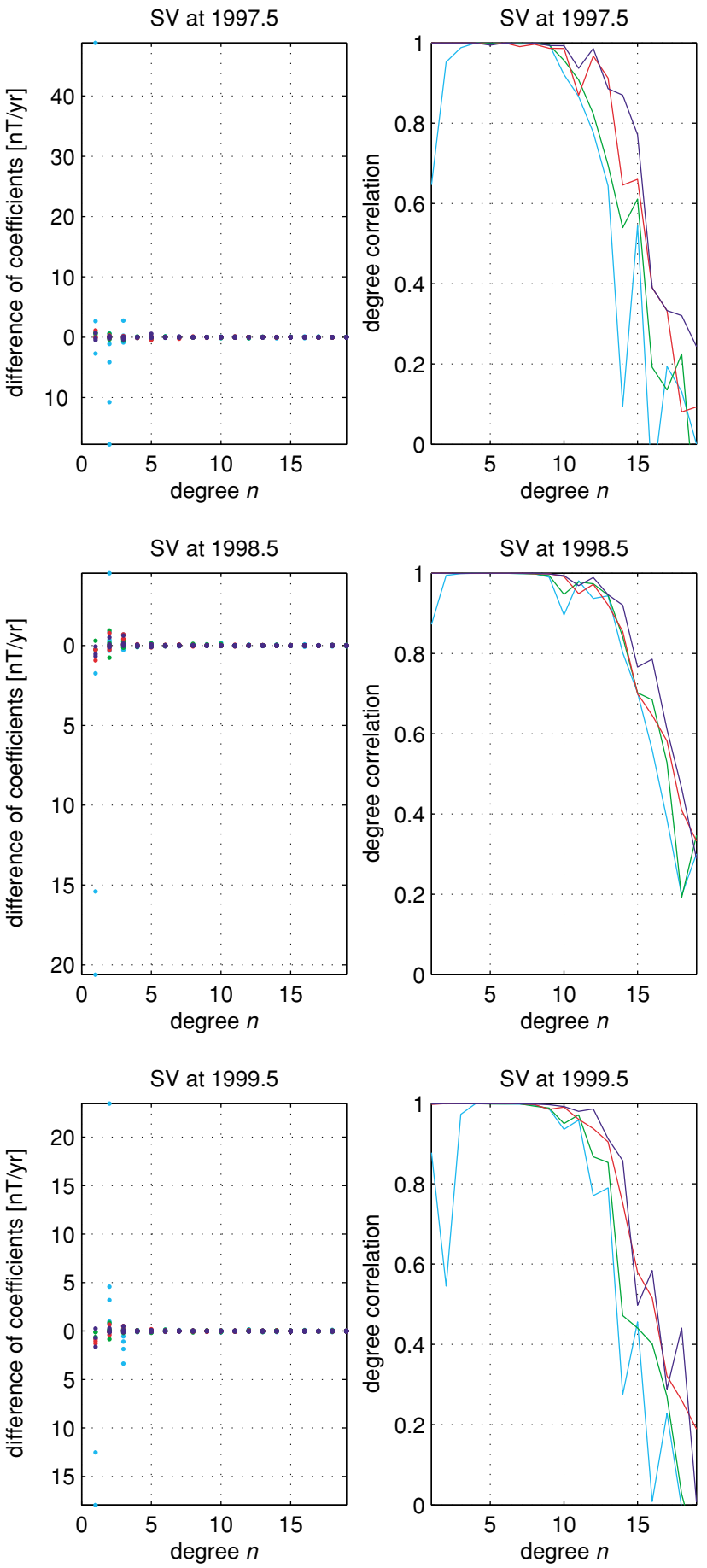

Fig. 5. Comparison of original and recovered secular variation (SV) at 1997.5, 1998.5 and 1999.5 at Earth's surface for each of the four combinations of satellites, SW-A, SW-AB, SW-ABC, and SW-ABCD. On the left are the $R_{n}$ spectra for the original (black) and for the difference between original and recovered SW-A (light blue), SW-AB (green), SW-ABC (red), and SW-ABCD (dark blue) models. In the middle are the differences in coefficients as a function of degree $n$ (same color scheme). On the right are the degree correlations, $\rho_{n}$, between the recovered and original models (same color scheme).

pansions defined by $N_{\max }=3$ and $M_{\mathrm{mxm}}=1$. However, the associated high-frequency induced signal is a function of 3-D conductivity structure and requires a more complicated spatial representation. In this case a $N_{\max }=3$ and $M_{\mathrm{mxi}}=3$ expansion is used. It will be seen that in order to resolve these features, one should have satellites in at least two well-separated orbit planes. This results in $L_{\mathrm{bi}}=15$ and $L_{\mathrm{bm}}=9$ for a total of $L_{\mathrm{b}}=24$ coefficients per $1 \mathrm{hr}$ bin. Recall that the number of bins having a sufficient amount of data for this parameterization is $\ell \approx 14,000$. Thus, the total number of magnetospheric and high-frequency induced coefficients is about 336,000; the grand total of parameters to be estimated is then $\operatorname{dim}(\mathbf{x}) \approx 358,360$. The number of linear orthogonality constraints is $K=90$.

The performance of this constellation in regards to SV and lithospheric recovery are indicated in Figs. 5, 6, and 7 in the form of comparisons between the recovered and original models. The quality of the high-degree SV recovery 

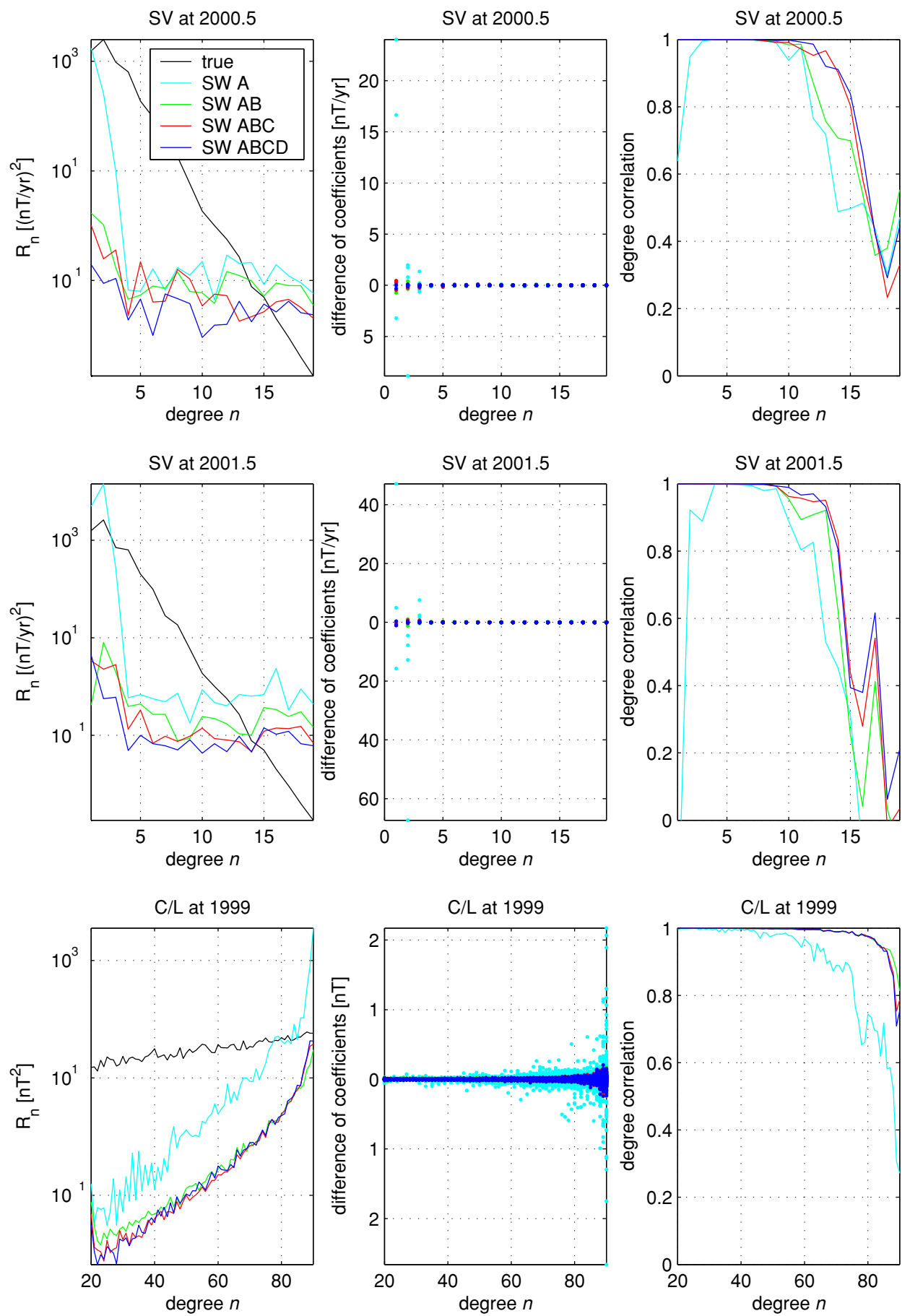

Fig. 6. Same as Fig. 5, but for secular variation (SV) at 2000.5 and 2001.5 and for the core and lithosphere (C/L) at 1999.0 at Earth's surface (bottom panels).

varies over the life of the mission, being a bit better towards the middle of the mission. Degrees 1-14 are recovered consistently above the 0.7 correlation threshold, but degrees up to 16 are recovered at this level during certain times. These values are also confirmed by the cross-over point of the $R_{n}$ spectra of the original and difference; the degree above which errors begin to dominate the signal. It is expected that recovery of degrees 1-3 will be enhanced if observatory data are used. The sensitivity matrices show a gradual degradation across all orders with increasing degree. It should be noted that power associated with any long-term trends existing in the magnetospheric induced contributions would necessarily be incorporated into that of the SV by design and could be the cause of the observed deteriorations at high degree.

The crustal field is being recovered adequately from degrees 20-90; the degree correlations are above 0.97 through degree 80 . The sensitivity matrix shows uniform recovery that is roughly independent of order and which very gradually deteriorates with increasing degree. Again, the "hemispherical" excursion pattern becomes prominent near $n=90$. 

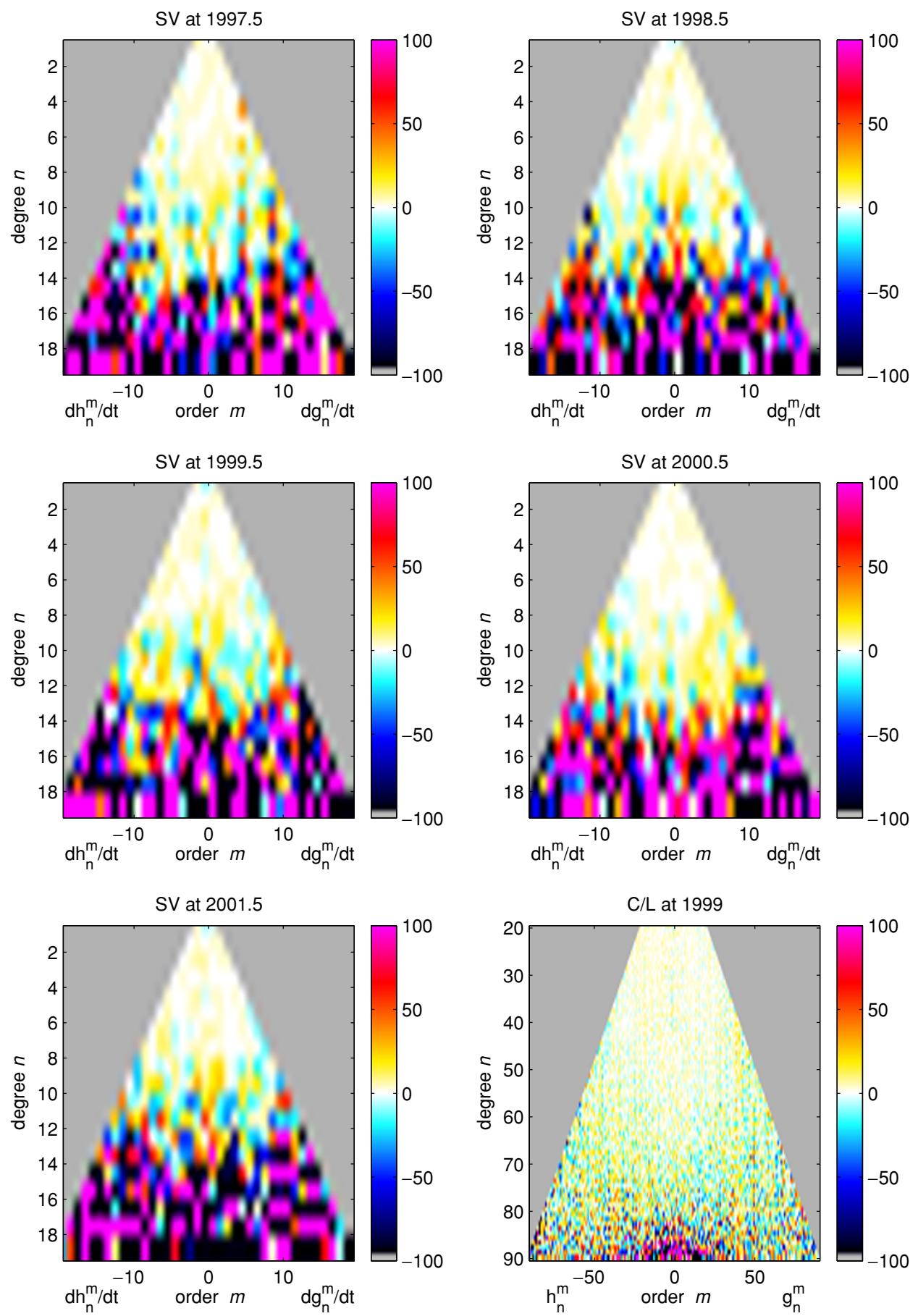

Fig. 7. Sensitivity matrices, $S(n, m)$, for the SW-ABCD secular variation (SV) and core and lithospheric (C/L) models corresponding to the epochs in Fig. 5.

The effects of orbital configuration on the recoverability of the SV and the time-series of magnetospheric and highfrequency induced contributions are illustrated in Figs. 811. The first figure shows the main field $g_{n}^{m}$ and $h_{n}^{m}$ coefficient estimates from SW-ABCD (green) and the original coefficients (dark blue) over the mission envelope for degrees $n=1-3$, and the second, third and fourth figures show the same for the SV $\dot{g}_{n}^{m}$ and $\dot{h}_{n}^{m}$, the magnetospheric $q_{n}^{m}$ and $s_{n}^{m}$, and the high-frequency induced $a_{n}^{m}$ and $b_{n}^{m}$ coefficients, respectively. Recall that the magnetospheric coefficients are only determined over orders $m=0-1$. These real coeffi- cients are related to the complex such that $\gamma_{n}^{m}=g_{n}^{m}-i h_{n}^{m}$, $\mu_{n}^{m}=q_{n}^{m}-i s_{n}^{m}$ and $\iota_{n}^{m}=a_{n}^{m}-i b_{n}^{m}$. Note that the geometries are slightly different here since the main field and $\mathrm{SV}$ coefficients relate to geographic coordinates while the magnetospheric and induced coefficients relate to dipole coordinates. The original magnetospheric and induced coefficients have been shifted by various amounts in order to facilitate visual comparisons.

In general, one sees fairly good recovery of the SV coefficients over the middle portion of the envelope, but degraded near the edges. This translates to good agreement in trend 

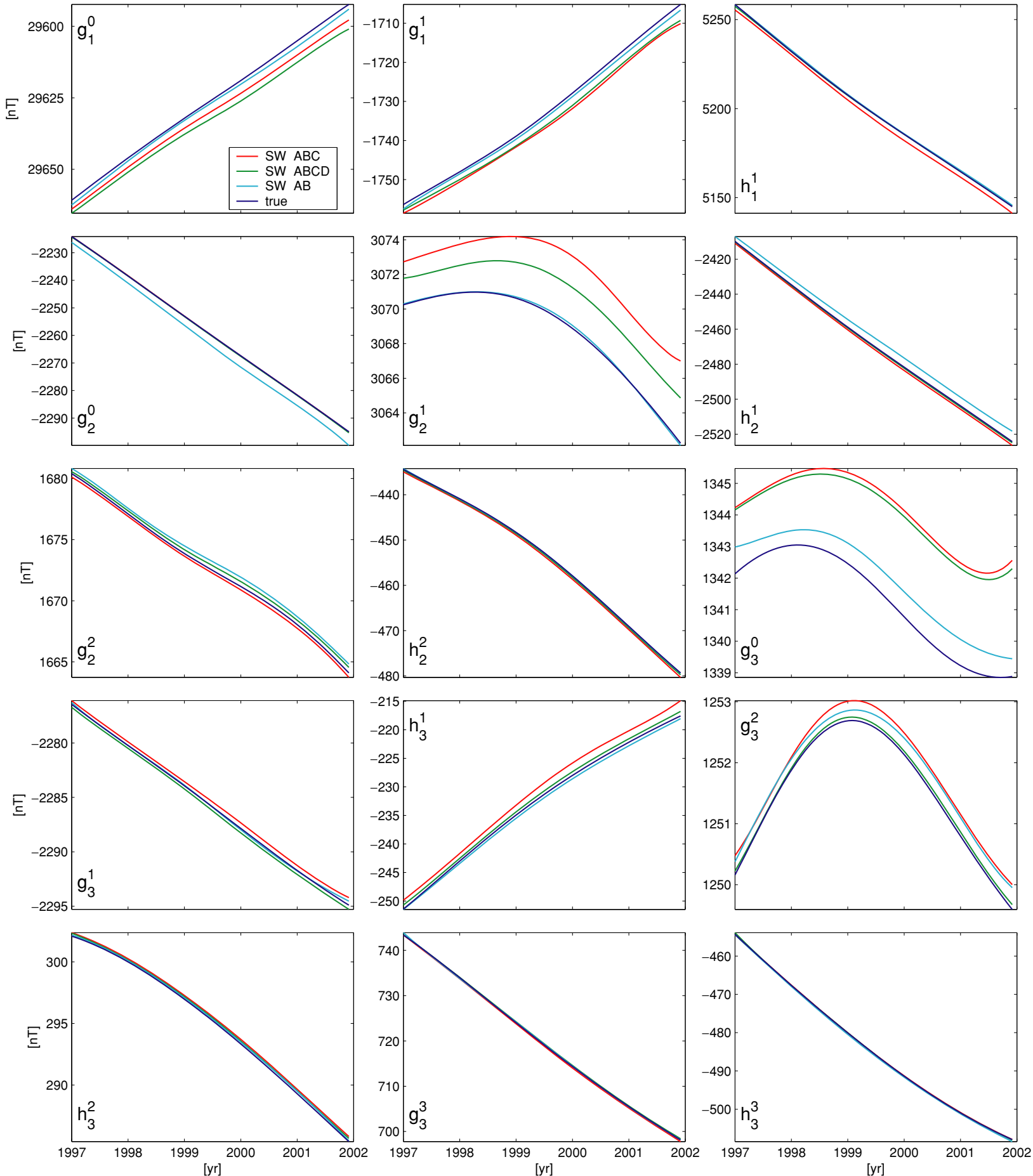

Fig. 8. Plots of the main field coefficients $g_{n}^{m}=\Re\left\{\gamma_{n}^{m}\right\}$ and $h_{n}^{m}=\mathfrak{\Im}\left\{\gamma_{n}^{m}\right\}$ for $n=1-3$ over the mission envelope derived from quiet-time data from SW-ABC (red), SW-ABCD (green) and SW-AB (light blue) compared to the true coefficients (dark blue).

for the main field coefficients. However, there are some noteworthy coefficients whose offset mismatches are large with respect to total SV over the mission, e.g, $g_{2}^{1}$ and $g_{3}^{0}$. These offsets could be due to ionospheric baseline leakage due to the absence of surface data, especially since these are low orders. There are also some cases in which simpler constellations perform better than SW-ABCD, and these will be discussed later.

Looking at the magnetospheric and high-frequency induced coefficients, one notices large oscillations in some of them before mid-1998, which, in the latter case, attenuate to a minimum amplitude around 2000 , and then slightly increase towards the end of the mission. Vertical dotted lines have been added to Figs. 10 and 11 to mark separations of 3, 6 , and $9 \mathrm{hr}$ in local time between the Swarm A and C orbital planes. These patterns appear to correlate extremely well with the evolution of the local-time separation between the orbit planes. Although one may be tempted to explain these as edge effects related to the SV B-spline basis functions, the stability of the SW-AB constellation solutions for the 

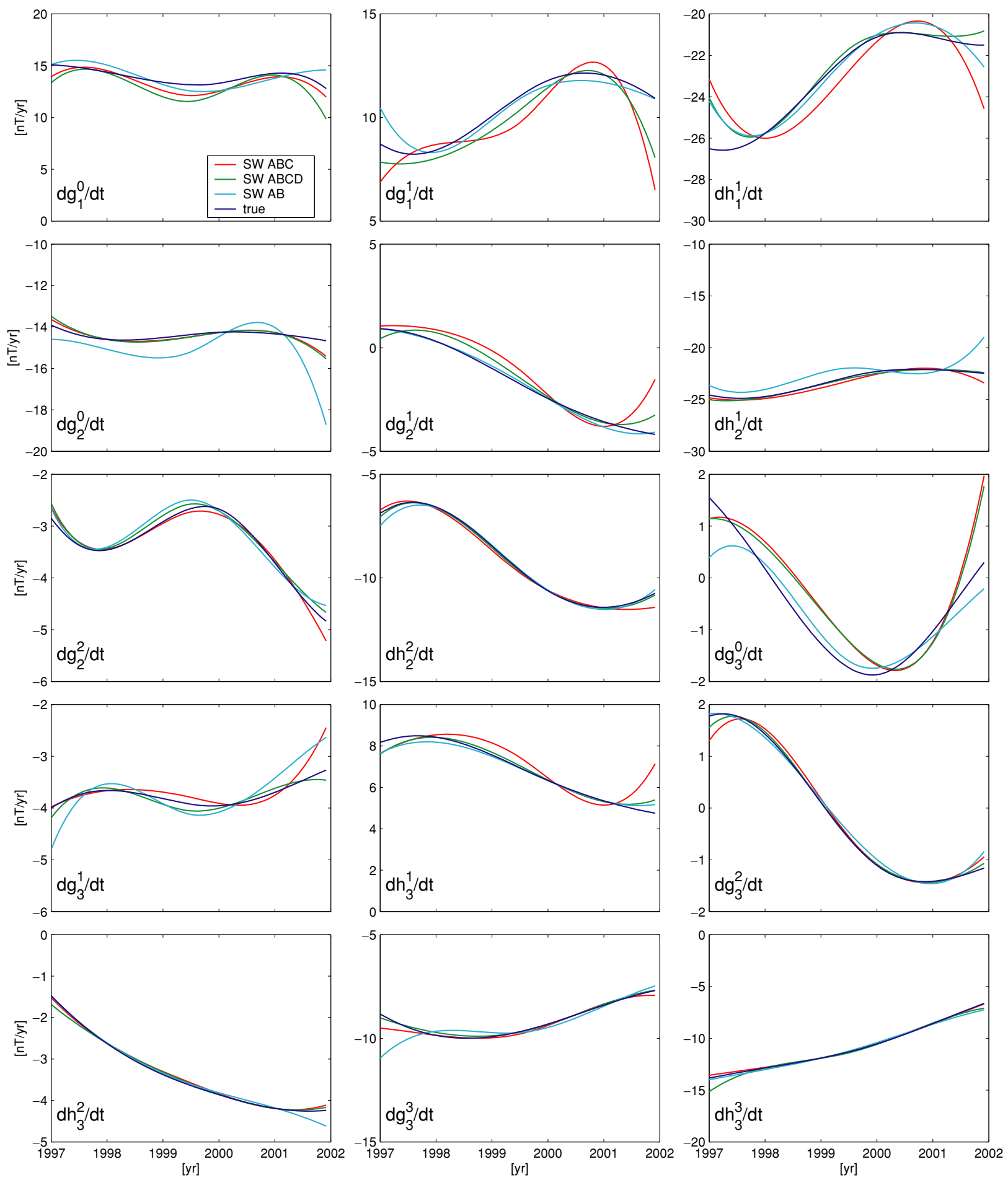

Fig. 9. Plots of the secular variation coefficients $\dot{g}_{n}^{m}=\Re\left\{\dot{\gamma}_{n}^{m}\right\}$ and $\dot{h}_{n}^{m}=\mathfrak{\Im}\left\{\dot{\gamma}_{n}^{m}\right\}$ for $n=1-3$ over the mission envelope derived from quiet-time data from SW-ABC (red), SW-ABCD (green) and SW-AB (light blue) compared to the true coefficients (dark blue).

high-frequency induced coefficients, to be discussed later, suggests otherwise.

Despite this, excellent recovery is seen in all of the magnetospheric and most of the high-frequency induced coefficients, particularly during the latter half of the mission and for lower order $m$. Table 2 lists the correlations between the true and recovered time-series for the entire mission (19972002) and the latter half of the mission (1999-2002) for each constellations considered in this study. In this context, the correlation is similiar to Eq. (35), except that the two vectors are the recovered and true signals whose elements are their values during each quiet-time bin. For SW-ABCD, one sees high correlations among the magnetospheric coefficients, which consistently increase for the latter half of the mission during which the orbital planes are more separated. As for the high-frequency induced coefficients, the correlations are generally much lower than for their magnetospheric counterpart. Indeed, they usually decrease with 

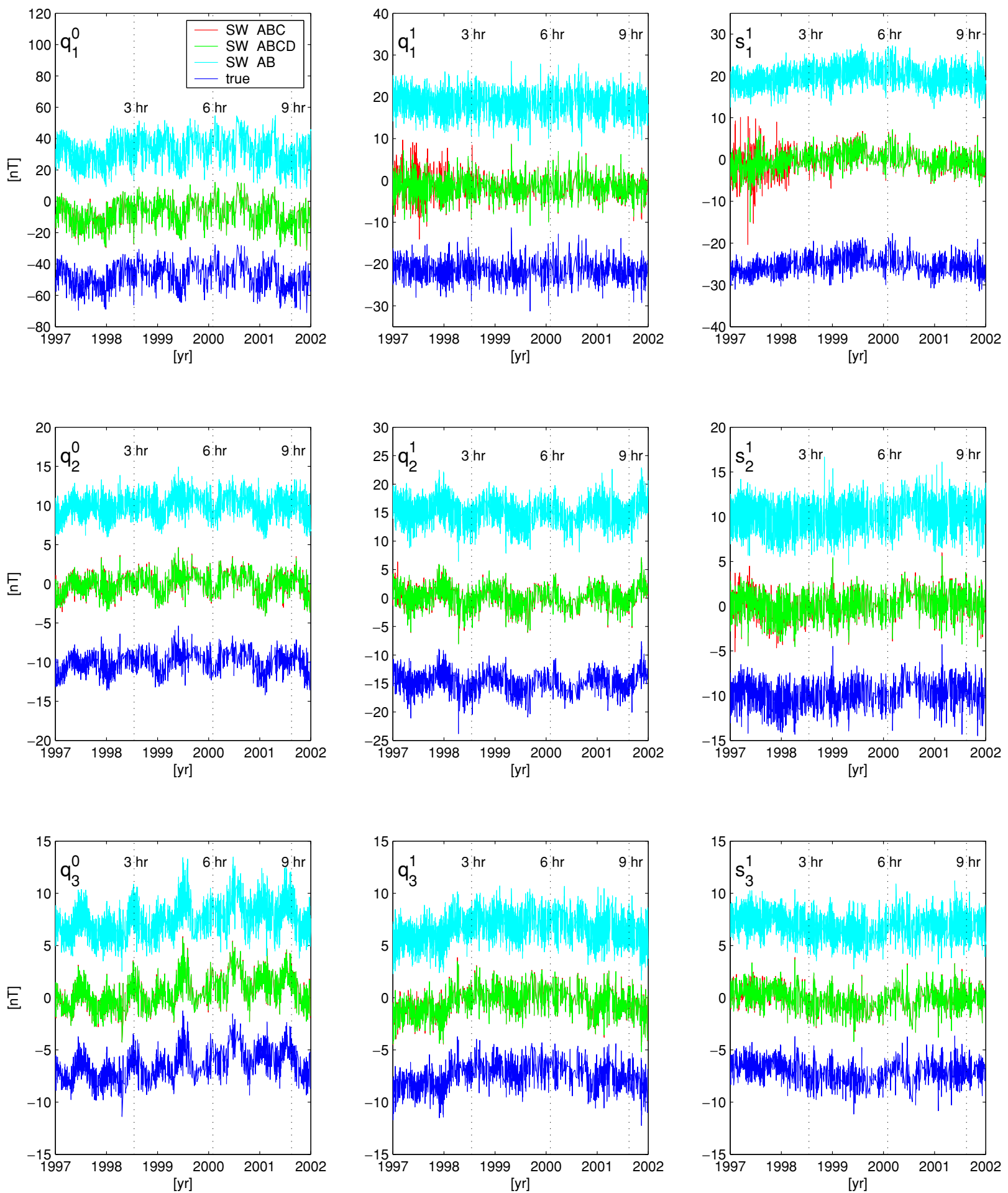

Fig. 10. Plots of the magnetospheric coefficients $q_{n}^{m}=\Re\left\{\mu_{n}^{m}\right\}$ and $s_{n}^{m}=\Im\left\{\mu_{n}^{m}\right\}$ for $n=1-3, m=0-1$ over the mission envelope derived from quiet-time data from SW-ABC (red), SW-ABCD (green) and SW-AB (light blue) compared to the true coefficients (dark blue). Vertical dotted lines mark separations of 3,6, and $9 \mathrm{hr}$ in local time between the Swarm A and C orbital planes; satellites are either co-rotating or counter-rotating for separations less than or greater than $6 \mathrm{hr}$, respectively. The SW-AB and true coefficients have been shifted in order to facilitate a visual comparison; the shifts for $q_{1}^{0}, q_{1}^{1}, \ldots, s_{3}^{1}$ are $40,20,20,10,15,10,7,7$ and $7 \mathrm{nT}$, respectively, for the SW-AB and $-40,-20,-25,-10,-15,-10,-7,-7$ and $-7 \mathrm{nT}$, respectively, for the true coefficients.

increasing $m$ and do generally increase with larger orbital plane separations seen after 1999. One should realize that while these correlations can be quite modest, they reflect all periods present in the series; it is expected that these values will fluctuate amongst individual periods. In addition, if the bin duration is increased to allow better spatial coverage, then these higher-order harmonics are recovered much better, at least below the Nyquist frequency (see Kuvshinov et al., 2006).

The influence of the orthogonality constraints on the 

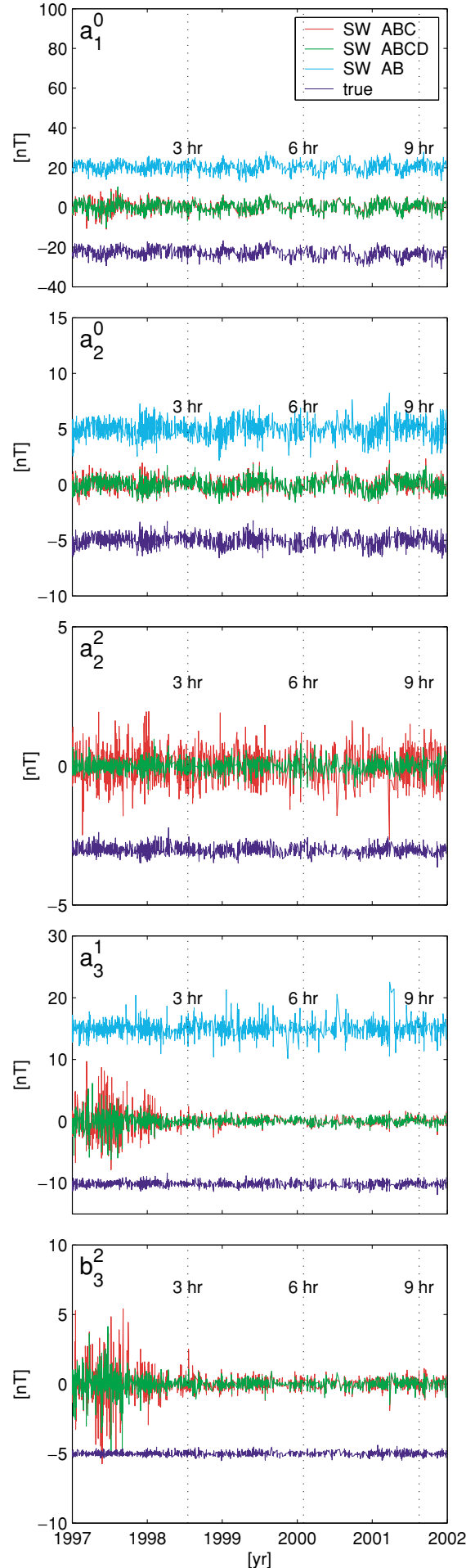
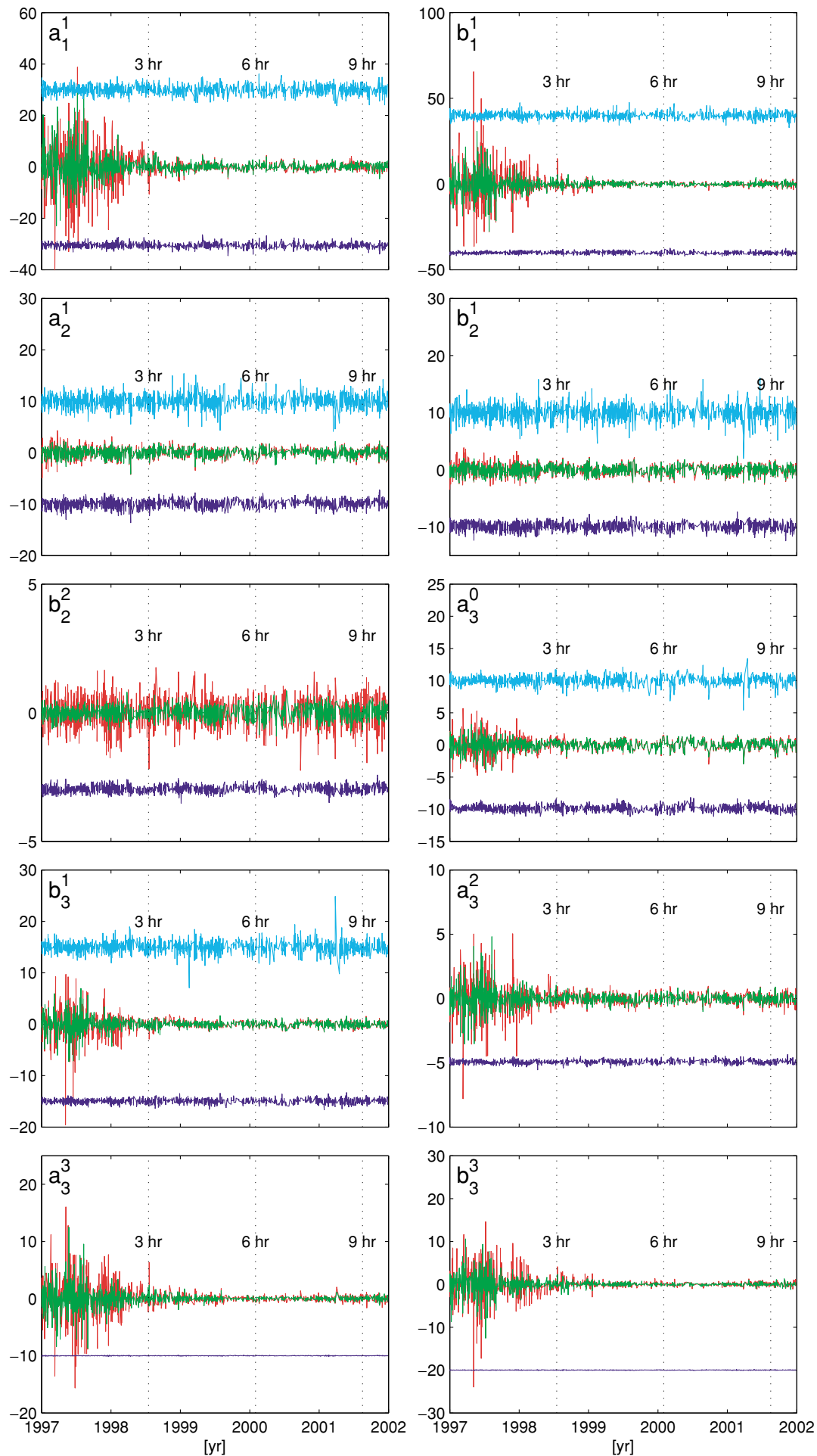

Fig. 11. Plots of the high-frequency induced coefficients $a_{n}^{m}=\Re\left\{\iota_{n}^{m}\right\}$ and $b_{n}^{m}=\Im\left\{\iota_{n}^{m}\right\}$ for $n=1-3$ over the mission envelope derived from quiet-time data from SW-ABC (red), SW-ABCD (green) and SW-AB (light blue) compared to the true coefficients (dark blue). Vertical dotted lines mark separations of 3, 6, and $9 \mathrm{hr}$ in local time between the Swarm A and C orbital planes; satellites are either co-rotating or counter-rotating for separations less than or greater than $6 \mathrm{hr}$, respectively. The SW-AB and true coefficients have been shifted in order to facilitate a visual comparison; the shifts for $a_{1}^{0}, a_{1}^{1}, \ldots, b_{3}^{3}$ are $20,30,40,5,10,10,10,15$, and $15 \mathrm{nT}$, respectively, for the SW-AB and $-20,-30,-40,-5,-10,-10,-3,-3$, $-10,-10,-15,-5,-5,-10$ and $-20 \mathrm{nT}$, respectively, for the true coefficients.

time-series of the recovered high-frequency induced coefficients can be seen in Fig. 11 where they all oscillate about the zero level (the true and SW-AB cases have been artificially offset for visual clarity), which is a consequence of being orthogonal to the SV base level; contrast this with the magnetospheric coefficients in Fig. 10 which are not so re- stricted. However, Olsen et al. (2005) argue that induced contributions should be zero-mean over appropriate periods such that this restriction is physically plausible. Inspection of the true induced coefficients in these plots indicates that they indeed oscillate about a near-zero level when the artificial offset is taken into account. 
Table 2. Correlations between true and recovered magnetospheric $\left(q_{n}^{m}\right.$ and $\left.s_{n}^{m}\right)$ and high-frequency induced $\left(a_{n}^{m}\right.$ and $\left.b_{n}^{m}\right)$ coefficient time-series derived from constellations SW-ABCD, SW-ABC and SW-AB calculated over the time spans 1997-2002 and 1999-2002.

SW-ABCD

1997-2002

1999-2002

\begin{tabular}{|c|c|c|c|c|c|c|c|c|c|}
\hline \multirow[b]{2}{*}{$\mathrm{n}$} & \multirow[b]{2}{*}{$\mathrm{m}$} & & & \multirow[b]{2}{*}{$b_{n}^{m}$} & \\
\hline & & $a_{n}^{m}$ & $q_{n}^{m}$ & $b_{n}^{m}$ & $s_{n}^{m}$ & $a_{n}^{m}$ & $q_{n}^{m}$ & & $s_{n}^{m}$ \\
\hline 1 & 0 & 0.52089 & 0.99960 & & & 0.50124 & 0.99981 & & \\
\hline 1 & 1 & 0.26071 & 0.94174 & 0.28911 & 0.88463 & 0.71630 & 0.99577 & 0.84385 & 0.99204 \\
\hline 2 & 0 & 0.90590 & 0.99714 & & & 0.89910 & 0.99769 & & \\
\hline 2 & 1 & 0.87873 & 0.99570 & 0.87833 & 0.99332 & 0.83874 & 0.99750 & 0.85650 & 0.99698 \\
\hline 2 & 2 & 0.45440 & - & 0.45744 & - & 0.34466 & - & 0.40358 & - \\
\hline 3 & 0 & 0.49713 & 0.99922 & & & 0.59019 & 0.99948 & & \\
\hline 3 & 1 & 0.37767 & 0.99676 & 0.35697 & 0.99215 & 0.77858 & 0.99854 & 0.85185 & 0.99801 \\
\hline 3 & 2 & 0.21078 & - & 0.28762 & - & 0.43964 & - & 0.60716 & - \\
\hline 3 & 3 & 0.04595 & - & 0.01398 & - & 0.08002 & - & 0.11341 & - \\
\hline
\end{tabular}

1997-2002

1999-2002

\begin{tabular}{|c|c|c|c|c|c|c|c|c|c|}
\hline \multirow[b]{2}{*}{$\mathrm{n}$} & \multirow[b]{2}{*}{$\mathrm{m}$} & & & & \multirow[b]{2}{*}{$s_{n}^{m}$} \\
\hline & & $a_{n}^{m}$ & $q_{n}^{m}$ & $b_{n}^{m}$ & $s_{n}^{m}$ & $a_{n}^{m}$ & $q_{n}^{m}$ & $b_{n}^{m}$ & \\
\hline 1 & 0 & 0.48771 & 0.99908 & & & 0.50038 & 0.99960 & & \\
\hline 1 & 1 & 0.22210 & 0.85373 & 0.22330 & 0.72983 & 0.63666 & 0.99117 & 0.68310 & 0.98354 \\
\hline 2 & 0 & 0.80750 & 0.98524 & & & 0.83928 & 0.98852 & & \\
\hline 2 & 1 & 0.77784 & 0.98071 & 0.75146 & 0.97199 & 0.80431 & 0.99200 & 0.80241 & 0.99082 \\
\hline 2 & 2 & 0.12894 & - & 0.21288 & - & 0.09530 & - & 0.15767 & - \\
\hline 3 & 0 & 0.41302 & 0.99764 & & & 0.57268 & 0.99840 & & \\
\hline 3 & 1 & 0.22396 & 0.99250 & 0.20702 & 0.98558 & 0.71814 & 0.99637 & 0.77091 & 0.99539 \\
\hline 3 & 2 & 0.14945 & - & 0.17354 & - & 0.32902 & - & 0.47520 & - \\
\hline 3 & 3 & -0.02989 & - & 0.00843 & - & 0.04276 & - & 0.06703 & - \\
\hline
\end{tabular}

1997-2002

1999-2002

\begin{tabular}{|c|c|c|c|c|c|c|c|c|c|}
\hline $\mathrm{n}$ & $\mathrm{m}$ & $a_{n}^{m}$ & $q_{n}^{m}$ & $b_{n}^{m}$ & $s_{n}^{m}$ & $a_{n}^{m}$ & $q_{n}^{m}$ & $b_{n}^{m}$ & $s_{n}^{m}$ \\
\hline 1 & 0 & 0.52190 & 0.99860 & & & 0.48494 & 0.99841 & & \\
\hline 1 & 1 & 0.44273 & 0.94150 & 0.40028 & 0.88620 & 0.40911 & 0.94102 & 0.38576 & 0.88825 \\
\hline 2 & 0 & 0.65788 & 0.96234 & & & 0.61189 & 0.96616 & & \\
\hline 2 & 1 & 0.52340 & 0.92861 & 0.50112 & 0.89402 & 0.42775 & 0.91093 & 0.41887 & 0.88248 \\
\hline 2 & 2 & - & - & - & - & - & - & - & - \\
\hline 3 & 0 & 0.61336 & 0.98957 & & & 0.54623 & 0.99110 & & \\
\hline 3 & 1 & 0.32168 & 0.90647 & 0.35988 & 0.85536 & 0.30101 & 0.86682 & 0.36068 & 0.83207 \\
\hline 3 & 2 & - & - & - & - & - & - & - & - \\
\hline 3 & 3 & - & - & - & - & - & - & - & - \\
\hline
\end{tabular}

4.2.2 Swarm A, B and C This case, denoted as SW$\mathrm{ABC}$, is identical to the previous except that data from Swarm satellite D, one of the high-flyers, has been removed; thus, $\operatorname{dim}(\mathbf{d})=7,107,867$. The salient difference is that there is now one less orbital plane during maximum separation, which is expected to somewhat degrade recovery performance. Again, Figs. 5 and 6 indicate that high-degree $\mathrm{SV}$ recovery behaves generally the best towards the middle of the mission, where it is commensurate with SW$\mathrm{ABCD}$, and is only marginally worse than SW-ABCD near the edges. The sensitivity matrices, shown in Fig. 12, are also very close to those of SW-ABCD. Evidently, the re- moval of Swarm D would not greatly impact the SV recovery phase of the mission, at least as suggested by these metrics. A closer inspection of individual coefficients for degrees $n=1-3$ in Figs. 8 and 9 reveals an overall similar behavior in SV with SW-ABCD (green) with perhaps a slight degradation for SW-ABC (red), particularly near the mission edges. Offsets in the main field coefficients are also mostly similar.

Likewise, the quality of the crustal field recovery is very close to that of SW-ABCD, and in fact, may be better over certain coefficient ranges, such as for $n>80$. The sensitivity matrix is almost indistinguishable from that of SW- 

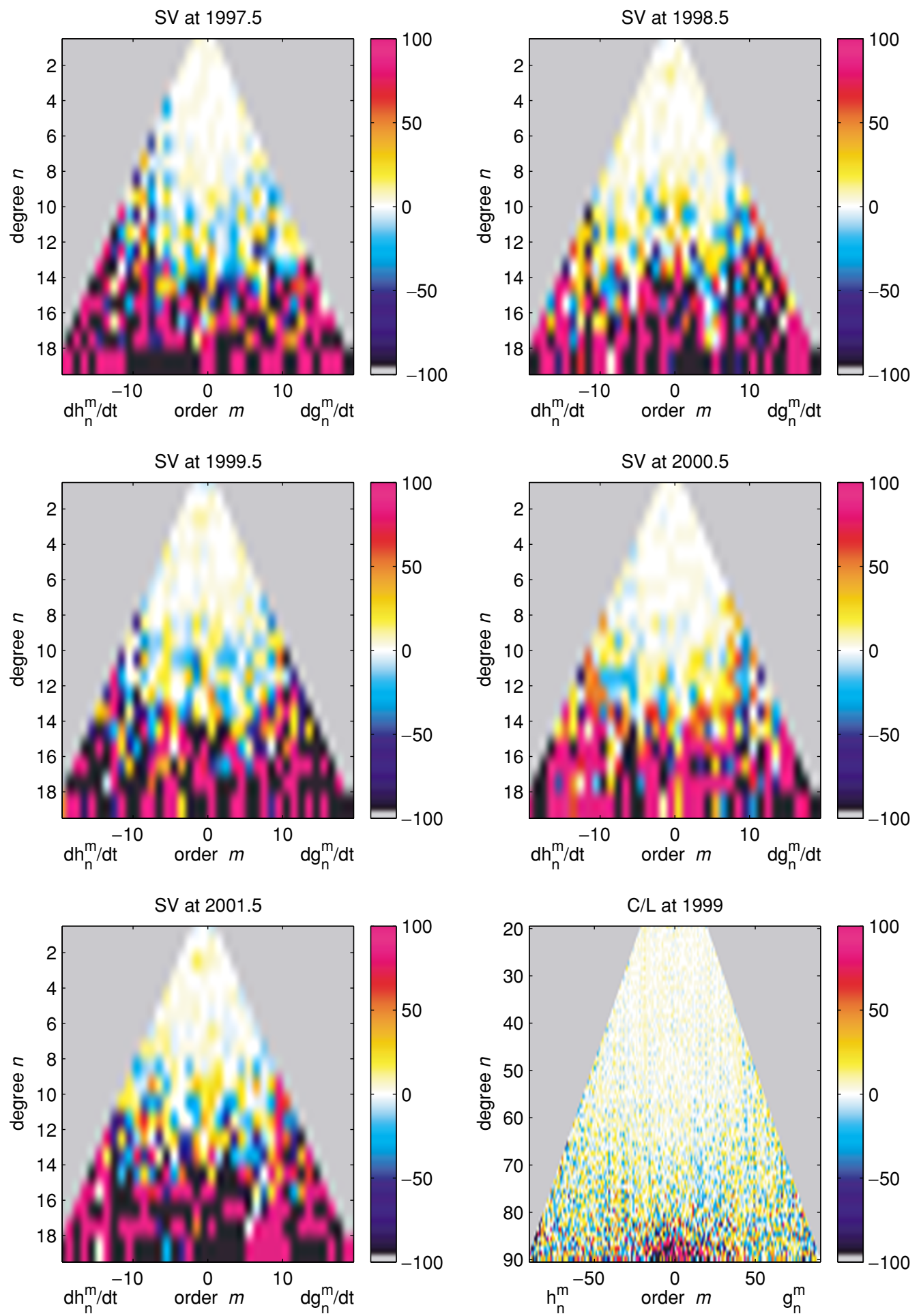

Fig. 12. Same as Fig. 7, but for the SW-ABC case.

$\mathrm{ABCD}$, which suggest that for lithospheric studies there is no compelling advantage of SW-ABCD over SW-ABC.

A more noticeable difference in performance between $\mathrm{SW}-\mathrm{ABCD}$ and SW-ABC is seen in the recovery of the magnetospheric and high-frequency induced time-series, although they are both quite comparable. Figure 10 shows that the SW-ABC (red) recovery of magnetospheric coefficients is nearly commensurate with SW-ABCD (green); only $q_{1}^{1}$ and $s_{1}^{1}$ exhibit much more oscillation in the SW-
$\mathrm{ABC}$ case, and this is only early in the mission. Table 2 supports this numerically and shows very little difference after 1999. Figure 11 shows similar plots for the highfrequency induced coefficients where SW-ABCD (green) and SW-ABC (red) performance is still rather close; of particular exception is the $a_{2}^{2}$ and $b_{2}^{2}$ pair, which is much more poorly recovered by SW-ABC. The most striking differences between the two cases are in the recovery of coefficients where large excursions occur before mid-1998 (the 
$a_{1}^{1}$ and $b_{1}^{1}$ pair and the $a_{3}^{m}$ and $b_{3}^{m}$ family) such that the amplitudes of the oscillations are significantly larger in the SW-ABC case. This suggests that the additional orbital plane of Swarm D is allowing for increased observability among the estimated magnetospheric and high-frequency induced parameters in a given bin. Evidently, the absence of the additional orbit plane in the SW-ABC case (though only $12^{\circ}$ separated Swarm C from D) will affect the modes differently and is most likely dependent upon order $m$, which is sensitive to the longitudinal sampling rate. Again, Table 2 supports these observations.

A closer look at the time-series for the $a_{1}^{1}$ and $b_{1}^{1}$ pair and the $a_{3}^{m}$ and $b_{3}^{m}$ family reveals a peculiar asymmetry in oscillation amplitudes with respect to the $6 \mathrm{hr}$ local time separation line: the amplitudes are generally much larger before this line than after. If the oscillations are amplified as a result of poor parameter observability, then intuitively, one would expect least amplitude where the orbits are most separated, i.e., at $6 \mathrm{hr}$ separation. If these amplifications were due only to that, then one would also expect symmetry about the $6 \mathrm{hr}$ line, i.e., separations at $3 \mathrm{hr}$ and $9 \mathrm{hr}$ are equivalent. Some of the apparent asymmetry is due to the fact that the $6 \mathrm{hr}$ line does not equally divide the mission envelope such that the smallest separation angles at the beginning of the mission have no counterparts later. These smallest separations will incite the largest oscillations because they do prohibit parameter observability. However, oscillations in the 2-3 hr separation segment are noticeably more spurious than their counterparts in the 9-10 hr segment, for example, in $b_{3}^{3}$.

Because the most likely scenario for launch involves orbital injection of all satellites from a single rocket, the ephemerides have been synthesized so that all satellites are initially co-rotating in approximately the same orbital plane. This means that they are co-rotating before and counter-rotating after the $6 \mathrm{hr}$ separation line, respectively. Now, if the field were completely static during a bin interval in which two satellites in the same orbital plane were sampling, then the estimation of the magnetospheric and high-frequency induced fields in that bin would not depend on whether the satellites were co-rotating or counterrotating since the solution to Eq. (25) is invariant to the rowordering in the residual vector, $\mathbf{d}-A \mathbf{x}$. Since there are no known drastic differences in the nature of the synthesized field between the co-rotation and counter-rotation phases, this asymmetry must be an effect of time variation in the field over the duration of a given bin.

One possibility follows from the fact that if the time variation in the field is represented by expanding each spherical harmonic coefficient in a Fourier time-series, and the coordinate system is then rotated such that its equatorial plane coincides with the orbital plane, then the field may be expanded on the circular orbits in terms of pairs of "travelling modes" of the form $\mathfrak{R}\{A \exp i(m \phi+k \omega t)\}$ and $\Re\{B \exp i(m \phi-k \omega t)\}$. The two modes propagate the same standing wave exp $i m \phi$ in opposite directions along the orbits with the same angular speed $k \omega$. The $A$ and $B$ are complex coefficients which are constant with respect to the orbital position angle, $\phi$. During the co-rotation phase, both satellites are moving with one mode, though at differ- ent speeds, and against the other; during counter-rotation, one satellite moves with while the other moves against a given mode. This opposing movement between satellite and mode, guaranteed during counter-rotation, is thought to smooth the signal more consistently across the bins than in the co-rotation phase in which the smoothing of one mode depends upon the differential speeds between it and the satellites and the satellite separation angle, $\Delta \phi$. Note that if differential speed is negligible, then phase-lock will occur and smoothing will depend on $\Delta \phi$, which is a function of orbital evolution, and thus, changes with time. Evidently, the smoothing process is complicated and depends upon many dynamic factors that change from bin to bin. However, whether arguing from the standpoint of multiple orbital planes in local time or from counter-rotating satellites, there is significant improvement in the recovery of all fields as a result of the constellation approach.

Until now, all but the ionospheric coefficients have been examined. Although these parameters may seem to affect the primary and secondary mission objectives only indirectly, they have a definite influence in the recoverability of the entire parameter state. In fact, they are likely to be the biggest challenge in properly determining the induced fields. Therefore, the ionospheric coefficients derived from the SW-ABC constellation will be compared with the true coefficients via a modified sensitivity matrix in which the $m$ summation limits of $\pm n$ in Eq. (37) are replaced by $\pm \min \left(n, M_{\max }\right)$, where $M_{\max }$ is the truncation level on QD order $m$. Since the true ionospheric model has anisotropic resolving power in QD latitude and longitude, being higher in the former to capture such features as the EEJ, this results in a QD truncation level that is much less for $m$ than for $n$. This drastically reduces, somewhat artificially, the size of the normalizing coefficient, and so only the non-trivial terms are considered here.

Plots of modified $S(n, m)$ matrices are shown in Fig. 13 where the plot columns correspond to either equinoxes or solstices and show the seasonal variation of the coefficients while the plot rows correspond to diurnal wavenumber $p$ and show the daily variation of the coefficients. The rows and columns of $S(n, m)$ correspond to quasi-dipole (QD) degree and order, respectively, with $d_{n}^{m}(p)=\Re\left\{\epsilon_{n p}^{m}\right\}$ and $c_{n}^{m}(p)=\Im\left\{\epsilon_{n p}^{m}\right\}$ where $\epsilon_{n p}^{m}=\epsilon_{n p}^{m}(s)$ evaluated at a particular season angle. Overall, the recovery appears to be very good. Most coefficients show less than 10-20\% error. The largest discrepancies are in the $p=0$ terms at the solstices and in the high degree $p=4$ terms. The former may very well be related to the absence of surface data which help resolve the static ionospheric baseline that resides in the $p=0$ terms. One may also notice alternating warm and cool colors along a given column corresponding to a particular $m$ value. These are likely the result of ringing in coefficients associated with high QD latitudinal resolution due to the lack of regularization in this model.

4.2.3 Swarm A and B In this case data from the two low-flying Swarm satellites, A and B, were used to derive a model designated SW-AB. Initially, the high-frequency induced field was modelled with an $N_{\max }=M_{\mathrm{mxi}}=3$ expansion, but this proved to be unobservable. Evidently, the orbit planes of these two satellites were not separated sufficiently 

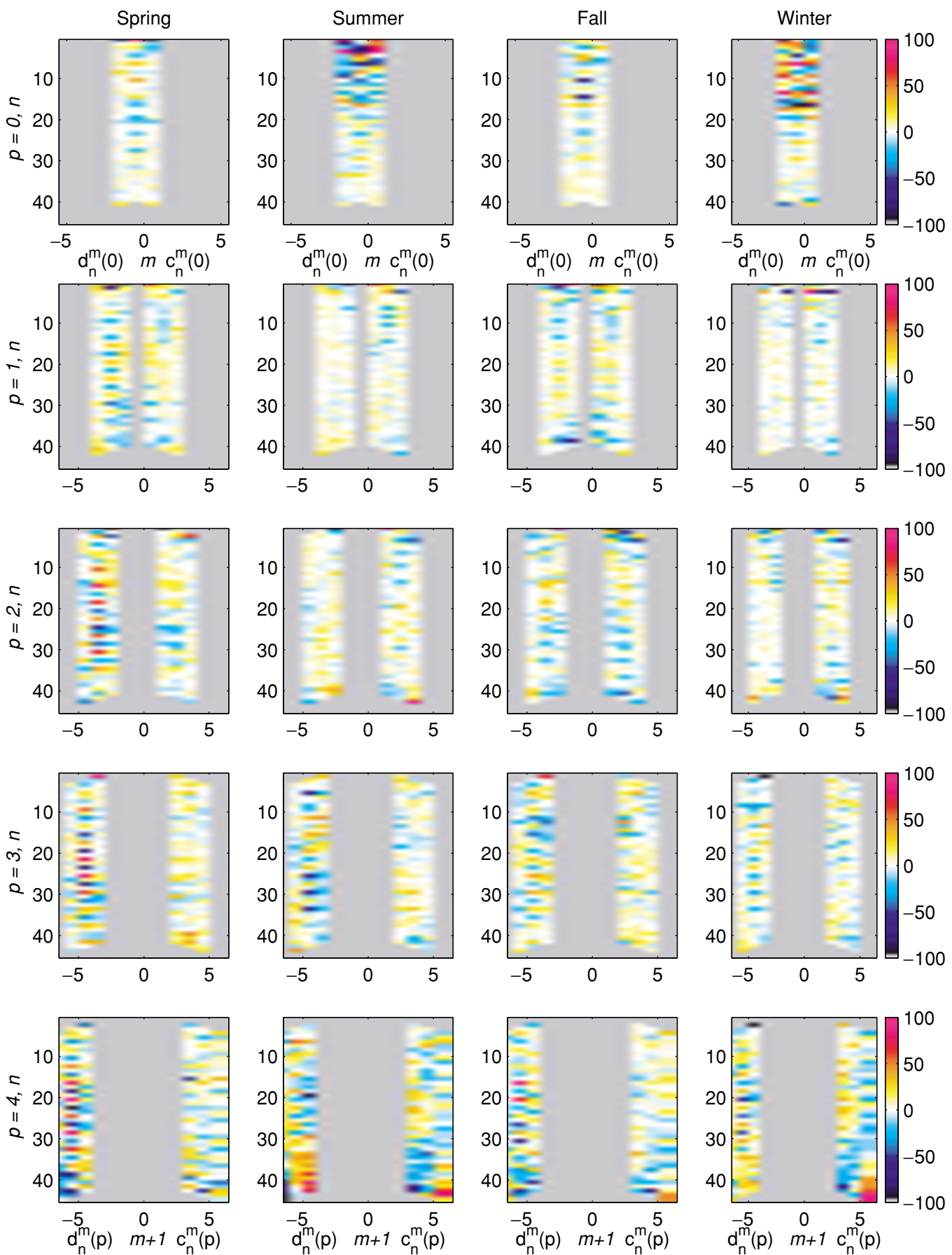

Fig. 13. Modified sensitivity matrices for the ionospheric model derived from quiet-time data from SW-ABC. The plot columns correspond to equinoxes and solstices and show the seasonal variation of the coefficients while the plot rows correspond to diurnal wavenumber $p$ and show the daily variation of the coefficients. The rows and columns of $S(n, m)$ correspond to quasi-dipole (QD) degree and order, respectively, with $d_{n}^{m}(p)=\Re\left\{\epsilon_{n p}^{m}\right\}$ and $c_{n}^{m}(p)=\Im\left\{\epsilon_{n p}^{m}\right\}$ where $\epsilon_{n p}^{m}=\epsilon_{n p}^{m}(s)$ evaluated at a particular season angle. However, note that while the columns of $S(n, m)$ correspond to QD order $m$ for $p=0$, they correspond to order $m+1$ for $p>0$. This allows both the real and imaginary parts of $\epsilon_{n p}^{0}$ to be accommodated in this schematic.

in local time. Thus, the final SW-AB model employed an $M_{\mathrm{mxi}}=1$, which was well resolved by this orbit configuration. Figures 5 and 6 show that there is a distinct improvement in SV recovery in both the SW-ABCD and SWABC models over SW-AB after about 1999, particularly for $n>11$. This corresponds quite well with the enhanced orbit separation between the high and low satellites by this time. In fact, the improvement is greater from SW-AB to
$\mathrm{SW}-\mathrm{ABC}$ then from SW-ABC to SW-ABCD. It is only for $n=1$ that $\mathrm{SW}-\mathrm{AB}$ shows some consistent improvement over the more complicated constellations, although 2000.5 is an exception. Again, it is expected that the addition of ground-based observatory data will help any of the constellations to resolve this properly. The sensitivity matrices do not differ greatly from the previous models and so they are not included. Figures 8 and 9 show that SW-AB (light blue) 
is in fact doing very well for $n=1-3$, in some cases much better than SW-ABC (red) and SW-ABCD (green).

As for crustal field recovery, SW-AB actually does a slightly better job than either SW-ABCD or SW-ABC at high degrees. This is most likely due to the fact that the high-altitude data from Swarm C and D are less sensitive to the high-degree crust, but still exert influence on the estimation. In practice, the weighting amongst the satellites should be tuned to reflect this effect.

The comparison of the time-series recovery of the magnetospheric coefficients for the SW-AB (light blue) case in Fig. 10 shows that it is of similar quality to that of the SW$\mathrm{ABC}$ (red) and SW-ABCD (green) cases; in fact, it is better than SW-ABC for $q_{1}^{1}$ and is the best for $s_{1}^{1}$ over the entire mission. It is markedly worse after 1999 as Table 2 confirms. Fig. 11 shows similar plots for the high-frequency induced coefficients and what is interesting is the apparent superior recovery of the $a_{1}^{1}$ and $b_{1}^{1}$ pair and $a_{3}^{0}$ prior to 1999 from the SW-AB (light blue) model; after 1999 SW-ABC and SW-ABCD are superior, in accordance with Table 2. This is a manifestation of the $M_{\mathrm{mxi}}=1$ truncation level used in SW-AB, which results in fewer parameters needing to be resolved. In the beginning of the mission the SW-ABC constellation offers no real advantage over SW-AB since the Swarm C is co-rotating in essentially the same plane as Swarm A and B. However, SW-ABC is being asked to resolve a much more complicated longitudinal structure in that $M_{\mathrm{mxi}}=3$. The addition of Swarm D at a $12^{\circ}$ separation angle in SW-ABCD is still not enough to initially resolve this higher $M_{\mathrm{mxi}}$. Although the magnetospheric truncation levels are identical amongst the three models, both the magnetospheric and the high-frequency induced fields are co-estimated, which leads to a wide spread observability problem as evidenced by the spurious oscillations in, for example, the $s_{1}^{1}$ time-series of SW-ABC. This suggests that future algorithms should incorporate some type of principle component analysis when modelling these fields in each bin.

4.2.4 Swarm A To complete the comparison, data from low-flying Swarm A were used to derive model SWA. As in the SW-AB case, truncation levels of $N_{\max }=3$ and $M_{\mathrm{mxm}}=M_{\mathrm{mxi}}=1$ were used in describing the magnetospheric and high-frequency induced fields in bins of $1 \mathrm{hr}$ duration. However, it was found that these fields, and consequently the SV field, were not well resolved. Therefore, the bin interval was increased to $3 \mathrm{hr}$ where the resulting observability was surprisingly good. Figures 5 and 6 show that the $R_{n}$ power in the discrepancies of the SV model remains consistently below the true signal power for $2<n<12$. There is severe degradation for $n>13$, as can be seen in the $\rho_{n}$ plots. The combination of spatial complexity and highfrequency variation present in the external and induced field contributions could not be resolved adequately with a single satellite. While increasing the bin duration allows more complicated spatial structure to be modelled, the longer averaging window evidently also allows unmodelled perturbations in the induced field to map into the high-degree SV.

The quality of the crustal field recovery can be deduced from Fig. 6. Only coefficients in the $n<78$ range have degree correlations above 0.7. Although not shown here, the sensitivity matrix indicates that much of the degradation is occurring when $n$ and $m$ are both above about 40. This is interesting since the problem of multiple satellite altitudes mentioned earlier does not apply here. It suggests that perhaps perturbations in the induced signal whose periods are shorter than the $3 \mathrm{hr}$ bin duration are contaminating the crustal field. Thus, the lack of multiple satellites in wellseparated orbits can be seen to degrade not only the SV recovery, but also that of the crustal field.

\subsection{Recovery from equally-incremented data}

Encouraged by the successful post-1999 recovery of the time-series of both the magnetospheric and high-frequency induced fields with the quiet-time restrictions, it was decided that an investigation should be undertaken to explore the feasibility of deducing conductivity structure directly from Swarm measurements. This involves the generation of a certain type of response function known as the " $C$ response", which may be interpreted as an inductive length scale for a particular frequency and position and is given by (Parkinson and Hutton, 1987)

$$
C(\omega, \mathbf{r})=\frac{B_{\mathrm{r}}(\omega, \mathbf{r})}{\nabla_{\mathrm{H}} \cdot \mathbf{B}_{\mathrm{H}}(\omega, \mathbf{r})},
$$

where $\omega$ is the frequency and $\nabla_{\mathrm{H}} \cdot \mathbf{B}_{\mathrm{H}}(\omega, \mathbf{r})$ is the horizontal divergence of the horizontal component of $\mathbf{B}(\omega, \mathbf{r})$. Because the derivation is done in the frequency domain, it requires Fourier analysis of the magnetic field in time and so is best achieved using uninterrupted time-series of the expansion coefficients at equal increments.

As alluded to earlier, the fidelity of the recovered magnetospheric and high-frequency induced time-series is highest when orbital plane separations are greatest and when the satellites are in counter-rotation phase. As a result, this exercise used data from the Swarm A, B and C satellites during the 1999-2002 time span at a continuous $1 \mathrm{~min}$ sampling rate, regardless of magnetic condition. Since a majority of the magnetic storm signature appears in the magnetospheric and associated induced field contributions, the methodology used here should suffice in discriminating most of the involved signals, especially since the data are synthesized by interpolating between $1 \mathrm{hr}$ snapshots of the underlying expansions of such perturbed fields (Olsen et al., 2006).

A three-satellite constellation was employed because, from previous arguments, this appears to be the minimum number required to meet the mission objectives stated so far. This yields 1,578,240 vector observations per satellite for a total measurement count of $\operatorname{dim}(\mathbf{d})=14,204,160$. Parameter, bin and orthogonality constraint counts for this case were quoted earlier in Section 3.2.1. However, because penetration of the induced fields is a function of frequency, with longer periods probing greater depths, the $C$-responses will also be sensitive to different depth ranges depending on their frequency. In order to resolve certain target features in the 3-D conductivity structure, this analysis was "tuned" to various frequencies by adjusting the bin duration; therefore, bins of 3, 6 and $12 \mathrm{hr}$ duration were also used. In these latter three cases, the longer bin durations allowed for more complicated internal field structure to be modelled, and so a slight modification was used in that Eqs. (18) and (19) 
were given different degree truncation levels: $N_{\max }=3$ and $M_{\mathrm{mxm}}=1$ for the magnetospheric field, but $N_{\max }=5$ and $M_{\mathrm{mxi}}=5$ for the high-frequency induced field.

Although the results of these cases are not included here, they are indeed commensurate with those of the quiet-time case. In fact, the quality of the time-series has allowed for the generation of intriguing $C$-response maps in a subsequent "mantle imaging" study, the details of which are reported in the companion paper of Kuvshinov et al. (2006). This study used the results from the $6 \mathrm{hr}$ bin case to successfully detect the presence of a large, hypothetical, regional conductor beneath the Pacific plate at a depth of 400$700 \mathrm{~km}$ from derived global $C$-responses having a 7.8 day period. These exciting results illustrate the enormous potential for scientific advancement in 3-D electromagnetic studies possible with the Swarm mission.

\section{Conclusions}

The combination of measurement acquisition through the Swarm constellation concept and analysis through the CI methodology has proven to be an effective means of achieving the Swarm mission objectives. It has been shown that a three-satellite constellation consisting of two lower spacecraft flying side-by-side in essentially a single orbital plane along with an additional higher spacecraft in a separate orbital plane, such that the planes precess at different rates, is sufficient in not only recovering the SV and lithospheric signal from noisy data over the course of the mission, but also time-series of magnetospheric and high-frequency induced fields. These time-series are of sufficient quality to allow realistic $C$-response functions to be derived, and thus, describe inductive length scales per frequency and position. These may subsequently be used to detect 3-D mantle conductivity structures, which themselves might be estimable directly from the data.

It is expected that if additional satellites were placed in distinct orbital planes, then even more complicated structure in the magnetosphere and associated induced fields could be discerned at discretization levels below $1 \mathrm{hr}$. In fact, early in this study a fine-scale time discretization was attempted with the ionospheric field and its associated induced contribution. However, the ionospheric field has much more small-scale structure than the magnetospheric field, especially on the dayside, e.g., the EEJ. Furthermore, it lies below the satellite sampling shells, thus requiring extensive surface data coverage in order to separate it from its induced counterpart. It may be possible though to exploit the regular time variability of this field, otherwise known as $\mathrm{Sq}$, such that realistic transfer functions may be developed which will allow these discretized snapshots to be applied.

Finally, the numerical methods employed in this study were sufficient in justifying the utility of the CI approach in treating the Swarm constellation data, but they could be improved upon. As mentioned earlier, parameter separability problems could be ameliorated by applying singular value decomposition (SVD) within each bin. This would allow the maximum amount of information to be extracted and would enhance overall recovery. In addition, alternatives to the rigid orthogonality constraints between the time variations of the induced and SV fields may be found which are more physically plausible; perhaps based upon longperiod induction responses. Clearly, much work remains to be done at all levels, but much progress has already been made.

Acknowledgments. We thank Richard Holme and Arnaud Chulliat for helpful reviews. The NASA Center for Computational Sciences at Goddard Space Flight Center provided computer support. TJS is supported under NASA Contract NAS5-00181.

\section{Appendix A. Length Comparisons Involving Pa- rameter State and Predicted Signal Vectors from Co- and Serial Estima- tion}

In this Appendix, the key Euclidean length relationships between the predicted signals given in Eq. (8) and parameter states given in Eq. (11) of Section 2 resulting from coestimation and serial estimation will be derived. The Euclidean $\ell_{2}$ norm of a general matrix $A$ is defined as (Demmel, 1997)

$$
|A| \equiv \sqrt{\lambda_{\max }\left(A^{*} A\right)},
$$

where $\lambda_{\max }(\cdot)$ denotes the largest eigenvalue and $*$ denotes the conjugate transpose. The following three key properties of the $\ell_{2}$ matrix norm will be needed, given real positive semi-definite (PSD) symmetric matrix $S$ and real general matrix $A$,

$$
\begin{aligned}
|S| & \equiv \lambda_{\max }(S) \\
\left|A^{-1}\right| & =\sqrt{\left(\lambda_{\min }\left(A A^{T}\right)\right)^{-1}} \\
|A| & =\left|A^{T}\right|
\end{aligned}
$$

where $\lambda_{\min }(\cdot)$ denotes the smallest eigenvalue, and of course, $\left|A^{-1}\right|=\infty$ for singular $A$ matrices.

The notation of Section 2 and its definitions of matrices $A$ and $B$ and vectors $\mathbf{d}$ and $\mathbf{x}_{B}$ will now be used throughout such that the following relationships hold

$$
\begin{aligned}
\mathcal{R}_{A} & =A\left(A^{T} A\right)^{-1} A^{T}, \\
\mathcal{N}_{A} & =I-\mathcal{R}_{A}, \\
\tilde{\mathbf{x}}_{B} & =\left(B^{T} \mathcal{N}_{A} B+B^{T} \mathcal{R}_{A} B\right)^{-1} B^{T} \mathcal{N}_{A} \mathbf{d}, \\
\mathbf{x}_{B} & =\left(B^{T} \mathcal{N}_{A} B\right)^{-1} B^{T} \mathcal{N}_{A} \mathbf{d}, \\
\tilde{\mathbf{d}}_{B} & =B \tilde{\mathbf{x}}_{B}, \\
\mathbf{d}_{B} & =B \mathbf{x}_{B} .
\end{aligned}
$$

First, assume that both $B^{T} \mathcal{N}_{A} B$ and $B^{T} B$ are invertible such that the Cholesky decompositions $B^{T} \mathcal{N}_{A} B=L L^{T}$ and $B^{T} B=K K^{T}$ exist, where $L$ and $K$ are lowertriangular matrices. This leads to

$$
\begin{aligned}
\left|\mathcal{N}_{A} \mathbf{d}_{B}\right| & =\left|\mathcal{N}_{A} B \mathbf{x}_{B}\right|, \\
& =\mathbf{x}_{B}^{T} B^{T} \mathcal{N}_{A} B \mathbf{x}_{B}, \\
& =\mathbf{x}_{B}^{T} L L^{T} \mathbf{x}_{B}, \\
& =\left|L^{T} \mathbf{x}_{B}\right|,
\end{aligned}
$$

and likewise

$$
\left|\mathbf{d}_{B}\right|=\left|K^{T} \mathbf{x}_{B}\right|
$$


The upper-bound on $\left|K^{-1} L\right|$ will also be needed in future calculations, and so by definition

$$
\left|K^{-1} L\right| \equiv \sqrt{\lambda_{\max }\left(L^{T} K^{-T} K^{-1} L\right)},
$$

but,

$$
\begin{aligned}
L^{T} K^{-T} K^{-1} L= & L^{T}\left(L L^{T}+B^{T} \mathcal{R}_{A} B\right)^{-1} L, \\
= & L^{T} L^{-T} \\
& \left(I+L^{-1} B^{T} \mathcal{R}_{A} B L^{-T}\right)^{-1} L^{-1} L, \\
= & \left(I+L^{-1} B^{T} \mathcal{R}_{A} B L^{-T}\right)^{-1}
\end{aligned}
$$

Since $L^{-1} B^{T} \mathcal{R}_{A} B L^{-T}$ is PSD, then $I+L^{-1} B^{T} \mathcal{R}_{A} B L^{-T}$ is positive definite (PD) symmetric with eigenvalues $\geq 1$, which leads to

$$
\left|K^{-1} L\right| \leq 1
$$

The second of the set of inequalities relating the lengths of various projections of the data signal vectors predicted by co-estimation, $\mathbf{d}_{B}$, and serial estimation, $\tilde{\mathbf{d}}_{B}$, in Eq. (8) is now straight forward to derive

$$
\begin{aligned}
\left|\tilde{\mathbf{d}}_{B}\right|= & \left|K^{T} \tilde{\mathbf{x}}_{B}\right| \\
= & \left|K^{T}\left(B^{T} B\right)^{-1} B^{T} \mathcal{N}_{A} \mathbf{d}\right| \\
= & \mid K^{T} K^{-T} K^{-1} L L^{T}\left(B^{T} \mathcal{N}_{A} B\right)^{-1} \\
& B^{T} \mathcal{N}_{A} \mathbf{d} \mid \\
\leq & \left|K^{-1} L\right| \mid L^{T}\left(B^{T} \mathcal{N}_{A} B\right)^{-1} \\
& B^{T} \mathcal{N}_{A} \mathbf{d} \mid \\
\leq & \left|L^{T} \mathbf{x}_{B}\right| \\
= & \left|\mathcal{N}_{A} \mathbf{d}_{B}\right|
\end{aligned}
$$

The first and last inequalities of Eq. (8) follow from the fact that since

$$
\left|\mathbf{d}_{B}\right|^{2}=\left|\mathcal{N}_{A} \mathbf{d}_{B}\right|^{2}+\left|\mathcal{R}_{A} \mathbf{d}_{B}\right|^{2},
$$

then

$$
\begin{aligned}
\left|\mathcal{N}_{A} \mathbf{d}_{B}\right|^{2} & \leq\left|\mathbf{d}_{B}\right|^{2}, \\
\left|\mathcal{N}_{A} \mathbf{d}_{B}\right| & \leq\left|\mathbf{d}_{B}\right| .
\end{aligned}
$$

Finally, it is interesting to note the following alternative derivation which relates the first and second inequalities of Eq. (8)

$$
\begin{aligned}
\left|\mathcal{N}_{A} \tilde{\mathbf{d}}_{B}\right|= & \left|L^{T} \tilde{\mathbf{x}}_{B}\right| \\
= & \left|L^{T} K^{-T} K^{-1} B^{T} \mathcal{N}_{A} \mathbf{d}\right| \\
= & \mid L^{T} K^{-T} K^{-1} L L^{T} L^{-T} L^{-1} \\
& B^{T} \mathcal{N}_{A} \mathbf{d} \mid \\
= & \left|L^{T} K^{-T} K^{-1} L L^{T} \mathbf{x}_{B}\right| \\
= & \left|L^{T} K^{-T} K^{-1} L \mathcal{N}_{A} \mathbf{d}_{B}\right| \\
\leq & \left|L^{T} K^{-T} K^{-1} L\right|\left|\mathcal{N}_{A} \mathbf{d}_{B}\right| \\
\leq & \left|\mathcal{N}_{A} \mathbf{d}_{B}\right|
\end{aligned}
$$

the last inequality following since $L^{T} K^{-T} K^{-1} L$ is PD symmetric with eigenvalues $\leq 1$.

Now, the upper-bound on the ratio of the lengths of $\tilde{\mathbf{x}}_{B}$ to $\mathbf{x}_{B}$ in Eq. (11) may be derived similarly as

$$
\begin{aligned}
\left|\tilde{\mathbf{x}}_{B}\right| & =\left|K^{-T} K^{-1} B^{T} \mathcal{N}_{A} \mathbf{d}\right| \\
& =\left|K^{-T} K^{-1} L L^{T} L^{-T} L^{-1} B^{T} \mathcal{N}_{A} \mathbf{d}\right| \\
& =\left|K^{-T} K^{-1} L L^{T} \mathbf{x}_{B}\right| \\
& =\left|L^{-T} L^{T} K^{-T} K^{-1} L L^{T} \mathbf{x}_{B}\right| \\
& \leq\left|L^{-T}\right|\left|L^{T} K^{-T} K^{-1} L\right|\left|L^{T}\right|\left|\mathbf{x}_{B}\right| \\
& \leq\left|L^{-T}\right|\left|L^{T}\right|\left|\mathbf{x}_{B}\right| \\
& =\left|L^{-1}\right|\left|L^{T}\right|\left|\mathbf{x}_{B}\right| \\
& =\sqrt{\frac{\lambda_{\max }\left(L L^{T}\right)}{\lambda_{\min }\left(L L^{T}\right)}}\left|\mathbf{x}_{B}\right| \\
& =\sqrt{\kappa\left(B^{T} \mathcal{N}_{A} B\right)}\left|\mathbf{x}_{B}\right|
\end{aligned}
$$

and so,

$$
\frac{\left|\tilde{\mathbf{x}}_{B}\right|}{\left|\mathbf{x}_{B}\right|} \leq \sqrt{\kappa\left(B^{T} \mathcal{N}_{A} B\right)},
$$

where Eq. (A.19) was used in going from Eq. (A.41) to Eq. (A.42) and where $\kappa\left(B^{T} \mathcal{N}_{A} B\right)$ is the condition number of $B^{T} \mathcal{N}_{A} B$, i.e., the ratio of the largest to smallest eigenvalues of $B^{T} \mathcal{N}_{A} B$ (see Demmel, 1997).

\section{References}

Arkani-Hamed, J. and D. W. Strangway, Intermediate-scale magnetic anomalies of the Earth, Geophysics, 50, 2817-2830, 1985a.

Arkani-Hamed, J. and D. W. Strangway, Lateral variations of apparent susceptibility of lithosphere deduced from Magsat data, J. Geophys. Res., 90, 2655-2664, 1985b.

Arkani-Hamed, J. and D. W. Strangway, Band-limited global scalar magnetic anomaly map of the Earth derived from Magsat data, J. Geophys. Res., 91, 8193-8203, 1986.

Arkani-Hamed, J., R. A. Langel, and M. E. Purucker, Magnetic anomaly maps of Earth derived from POGO and Magsat data, J. Geophys. Res., 99, 24,075-24,090, 1994.

Bertsekas, D. P., Nonlinear Programming, Athena Scientific, Belmont, MA, 1995.

Cohen, Y. and J. Achache, New global vector magnetic anomaly maps derived from Magsat data, J. Geophys. Res., 95, 10,783-10,800, 1990.

Cohen-Tannoudji, C., B. Diu, and F. Laloë, Quantum mechanics, Volume I, J. Wiley \& Sons, New York, 1977.

Counil, J., Y. Cohen, and J. Achache, The global continent-ocean magnetization contrast, Earth Planet. Sci. Lett., 103, 354-364, 1991.

Demmel, J. W., Applied numerical linear algebra, SIAM, Philadelphia, 1997.

Friis-Christensen, E., H. Lühr, and G. Hulot, Swarm: A constellation to study the Earth's magnetic field, Earth Planets Space, 58, this issue, 351-358, 2006.

Golub, G. H. and C. F. Van Loan, Matrix Computations, Johns Hopkins Univ. Press, Baltimore, 1989.

Green, A. A., A comparison of adjustment procedures for leveling aeromagnetic survey data, Geophysics, 48, 745-753, 1983.

Hamoudi, M., Y. Cohen, and J. Achache, Can the thermal thickness of the continental lithosphere be estimated from Magsat data?, Tectonophys., 284, 19-29, 1998.

Kuvshinov, A., T. Sabaka, N. Olsen, 3-D electromagnetic induction studies using the Swarm constellation: Mapping conductivity anomalies in the Earth's mantle, Earth, Planets and Space, 58, this issue, 417-427, 2006. Langel, R. A., The Main Field, in wGeomagnetism, vol. 1, edited by J. A. Jacobs, pp. 249-512, Academic Press, London, 1987.

Luyendyk, A. P. J., Processing of airborne magnetic data, J. Austr. Geol. Geophys., 17(2), 31-38, 1997. 
Maus, S., M. Rother, R. Holme, H. Lühr, N. Olsen, and V. Haak, First scalar magnetic anomaly map from CHAMP satellite data indicates weak lithospheric field, Geophys. Res. Lett., 29(14), doi: 10.1029/2001GL013,685, 2002.

Maus, S., H. Lühr, and M. Purucker, Simulation of the high-degree lithospheric field recovery for the Swarm constellation of satellites, Earth Planets Space, 58, this issue, 397-407, 2006.

Minty, B. R. S., Simple micro-levelling for aeromagnetic data, Expl. Geophys., 22, 591-592, 1991.

Olsen, N., The electrical conductivity of the mantle beneath Europe derived from $C$-Responses from 3 h to 720 h, Geophys. J., 133, 298-308, 1998.

Olsen, N., F. Lowes, and T. J. Sabaka, Ionospheric and induced field leakage in geomagnetic field models, and derivation of candidate models for DGRF 1995 and DGRF 2000, Earth Planets Space, 57, 1191-1196, 2005.

Olsen, N., R. Haagmans, T. J. Sabaka, A. Kuvshinov, S. Maus, M. E. Purucker, M. Rother, V. Lesur, and M. Mandea, The Swarm End-to-End mission simulator study: A demonstration of separating the various contributions to Earth's magnetic field using synthetic data, Earth Planets Space, 58, this issue, 359-370, 2006.

Parkinson, W. D. and V. R. S. Hutton, The Electrical Conductivity of the
Earth, in Geomagnetism, vol. 3, edited by J. A. Jacobs, pp. 261-321, Academic Press, London, 1987.

Ravat, D. N., R. A. Langel, M. E. Purucker, J. Arkani-Hamed, and D. E. Alsdorf, Global vector and scalar Magsat magnetic anomaly maps, $J$. Geophys. Res., 100, 20,111-20,135, 1995.

Richmond, A. D., Ionospheric Electrodynamics Using Magnetic Apex Coordinates, J. Geomagn. Geoelectr., 47, 191-212, 1995.

Sabaka, T. J., N. Olsen, and R. A. Langel, A comprehensive model of the quiet-time, near-Earth magnetic field: phase 3, Geophys. J. Int., 151, 32-68, 2002.

Sabaka, T. J., N. Olsen, and M. E. Purucker, Extending comprehensive models of the Earth's magnetic field with Ørsted and CHAMP data, Geophys. J. Int., 159, 521-547, doi: 10.1111/j.1365246X.2004.02,421.x, 2004.

Sorenson, H., Parameter Estimation, Marcel Dekker, Inc., New York, 1980.

Toutenburg, H., Prior Information in Linear Models, J. Wiley \& Sons, New York, 1982.

T. J. Sabaka (e-mail: sabaka@geomag.gsfc.nasa.gov) and N. Olsen 